JOURNAL OF THE

AMERICAN MATHEMATICAL SOCIETY

Volume 20, Number 1, January 2007, Pages 99-148

S 0894-0347(06)00546-7

Article electronically published on September 5, 2006

\title{
UNIVERSALLY OPTIMAL DISTRIBUTION OF POINTS ON SPHERES
}

\author{
HENRY COHN AND ABHINAV KUMAR
}

\section{Contents}

1. Introduction

2. Background

2.1. Hermite interpolation

2.2. Positive-definite kernels

3. Orthogonal polynomials

4. Linear programming bounds

5. Choosing auxiliary functions

6. Proof of the main theorem

7. The 600-cell

7.1. Proof that $h$ is positive definite

7.2. Proof that $h(t) \leq a(t)$

7.3. Proof of uniqueness

8. Other compact two-point homogeneous spaces

9. The Euclidean case

Acknowledgements

Appendix A. Uniqueness of spherical codes

References

\section{INTRODUCTION}

There are many beautiful and important configurations of points on the surface of a sphere, ranging from familiar examples such as the vertices of an icosahedron to more subtle cases such as the minimal vectors of the $E_{8}$ or Leech lattices. Yudin, Kolushov, and Andreev have shown in Y, KY1, KY2, An1, An2 that certain configurations, including the ones mentioned above, minimize specific sorts of energy. In this paper, we prove that in fact they minimize a far broader class of energies than was previously known; we call this property universal optimality (the precise definition is given below). Our proofs apply to a number of other configurations,

Received by the editors November 1, 2004.

2000 Mathematics Subject Classification. Primary 52A40, 52C17; Secondary 41A05.

Key words and phrases. Potential energy minimization, spherical codes, spherical designs, sphere packing.

The second author was supported by a summer internship in the Theory Group at Microsoft Research and a Putnam Fellowship at Harvard University.

(C) 2006 American Mathematical Society
Reverts to public domain 28 years from publication 
enumerated in Table 1 below, as well as to energy minimization in other compact two-point homogeneous spaces or Euclidean space.

The setting of this paper is potential energy minimization. If one constrains some identically charged particles to move on the surface of a sphere, how will they arrange themselves? They will eventually spread out so as to locally minimize their electrical potential energy, assuming their kinetic energy dissipates according to a force such as viscosity. We will ignore questions of dynamics and simply focus on the relative positions of the particles once they reach a minimum for potential energy. Furthermore, we will focus on global minima, although particles can indeed become trapped in a local minimum.

This question leads to a fundamental problem in extremal geometry, in which we abstract the key points by working in an arbitrary dimension and replacing the electrical potential with an arbitrary radial potential function. Let $f:(0,4] \rightarrow$ $[0, \infty)$ be any decreasing, continuous function. Given a finite subset $\mathcal{C}$ of the unit sphere $S^{n-1} \subset \mathbb{R}^{n}$, define the potential energy (more precisely, the $f$-potential energy) of $\mathcal{C}$ to be

$$
\sum_{x, y \in \mathcal{C}, x \neq y} f\left(|x-y|^{2}\right) .
$$

Because each pair $x, y$ is counted in both orders, our potential energy is twice that from physics. We normalize it this way because it makes the formulas prettier. It is also important to note that we view potential functions as functions of squared distance.

For a fixed number $N>1$, how can one choose $\mathcal{C}$ with $|\mathcal{C}|=N$ so as to minimize the potential energy? (To ensure that a minimum exists, when $\lim _{r \rightarrow 0+} f(r)$ is finite, we must allow degenerations in which points in the configuration coincide.) In most cases the answer is some complicated configuration, but it is remarkable how many important structures minimize potential energy.

In $\mathbb{R}^{3}$ the electrical potential corresponds to $f(r)=1 / r^{1 / 2}$, and a natural generalization to $\mathbb{R}^{n}$ with $n \geq 3$ is $f(r)=1 / r^{n / 2-1}$. That is an especially important power law, because $x \mapsto 1 /|x|^{n-2}$ is a harmonic function on $\mathbb{R}^{n} \backslash\{0\}$. However, any power law is of interest, as are more general potentials.

Many researchers have studied potential energy minima. One of the most beautiful rigorous approaches is due to Yudin in [Y]. He studied the harmonic power law and used spherical harmonics to give sharp lower bounds for potential energy for $n+1$ or $2 n$ points on $S^{n-1}$ (the optima are the regular simplex and cross polytope). This work was extended by Kolushov and Yudin in [KY1]. For $(n, N)=(8,240)$, they showed that the minimal vectors in the $E_{8}$ root lattice are the unique global minimum. Andreev applied their techniques in An2, An1 to show that the Leech lattice minimal vectors are the unique minimum for $(n, N)=(24,196560)$ and the vertices of the regular icosahedron are the unique minimum for $(n, N)=(3,12)$. In the present paper, we extend this approach to much more general potential functions, as well as numerous other point configurations (listed in Table 1).

The paper KY2 analyzed arbitrary power laws for the simplex, cross polytope, and $E_{8}$ root system in a closely related maximization problem (maximizing potential energy given an increasing potential function) and raised the question of how generally the techniques could be applied. We will deal with quite general potential functions. Without loss of generality one can assume that the potential function is nonnegative (only distances between 0 and 2 occur on the sphere, and one can 
add a constant to ensure nonnegativity over that range). The potential function must be decreasing, which corresponds to a repulsive force, and it is natural to require convexity as well. Our results require a strengthening of these conditions, namely complete monotonicity. Recall that a $C^{\infty}$ function $f: I \rightarrow \mathbb{R}$ on an interval $I$ is completely monotonic if $(-1)^{k} f^{(k)}(x) \geq 0$ for all $x \in I$ and all $k \geq 0$ (see [Wid, p. 145]) and strictly completely monotonic if strict inequality always holds in the interior of $I$. Of course, derivatives at endpoints of intervals denote one-sided derivatives. In this paper, endpoints are irrelevant, because we use halfopen intervals closed on the right, and one can show using the mean value theorem that complete monotonicity on the interior implies that it holds also at the right endpoint.

If a completely monotonic function fails to be strictly completely monotonic, then it must be a polynomial: if $f^{(k)}(x)=0$, then complete monotonicity implies that $f^{(k)}(y)=0$ for all $y>x$, in which case $f^{(k)}$ is identically zero because it is analytic (Theorem 3a in [Wid, p. 146]).

All inverse power laws $f(r)=1 / r^{s}$ with $s>0$ are strictly completely monotonic on $(0, \infty)$, and they are the most important cases for our purposes. Other examples include $f(r)=e^{-c r}$ with $c>0$.

It might seem preferable to use completely monotonic functions of distance, rather than squared distance, but squared distance is in fact more natural than it first appears. It simplifies formulas appearing in later sections of this paper, it fits into the general framework described in Section 8 , and it is more general than using distance: it is not hard to prove that if $r \mapsto f\left(r^{2}\right)$ is completely monotonic on a subinterval $(a, b)$ of $(0, \infty)$, then $f$ is completely monotonic on $\left(a^{2}, b^{2}\right)$, but not vice versa.

We will examine certain special configurations of points on high-dimensional spheres, which were introduced by Levenshtein in Lev2] (in which he proved that they are optimal spherical codes). We refer to them as the sharp arrangements, because everything fits into place so perfectly that many bounds turn out to be sharp. Each is a spherical code with certain parameters: an $(n, N, t)$ spherical code is a set of $N$ points on $S^{n-1}$ such that no two of them have inner product greater than $t$ (unless they are equal). In other words, all angles between them are at least $\cos ^{-1} t$. Each sharp configuration is also a spherical $M$-design for some $M$; recall that a spherical $M$-design is a finite subset of the sphere such that every polynomial on $\mathbb{R}^{n}$ of total degree at most $M$ has the same average over the design as over the entire sphere.

Definition 1.1. A finite subset of the unit sphere $S^{n-1}$ is a sharp configuration if there are $m$ inner products between distinct points in it and it is a spherical $(2 m-1)$-design.

Replacing $2 m-1$ with $2 m+1$ in Definition 1.1 would be impossible, for the following reason. If $t_{1}, \ldots, t_{m}$ are the inner products that occur between distinct points in a configuration and $y$ is a point in the configuration, then the polynomial

$$
x \mapsto(1-\langle x, y\rangle) \prod_{i=1}^{m}\left(\langle x, y\rangle-t_{i}\right)^{2}
$$

of degree $2 m+1$ vanishes on the entire configuration. However, its integral over the sphere does not vanish, because the polynomial is nonnegative on the sphere and not identically zero. Thus, sharp configurations are spherical designs of nearly the 
TABLE 1. The known sharp configurations, together with the 600-cell.

\begin{tabular}{|c|c|c|c|c|}
\hline$n$ & $N$ & $M$ & Inner products & Name \\
\hline 2 & $N$ & $N-1$ & $\cos (2 \pi j / N)(1 \leq j \leq N / 2)$ & $N$-gon \\
\hline$n$ & $N \leq n$ & 1 & $-1 /(N-1)$ & simplex \\
\hline$n$ & $n+1$ & 2 & $-1 / n$ & simplex \\
\hline$n$ & $2 n$ & 3 & $-1,0$ & cross polytope \\
\hline 3 & 12 & 5 & $-1, \pm 1 / \sqrt{5}$ & icosahedron \\
\hline 4 & 120 & 11 & $-1, \pm 1 / 2,0,( \pm 1 \pm \sqrt{5}) / 4$ & 600-cell \\
\hline 8 & 240 & 7 & $-1, \pm 1 / 2,0$ & $E_{8}$ roots \\
\hline 7 & 56 & 5 & $-1, \pm 1 / 3$ & kissing \\
\hline 6 & 27 & 4 & $-1 / 2,1 / 4$ & kissing/Schläfli \\
\hline 5 & 16 & 3 & $-3 / 5,1 / 5$ & kissing \\
\hline 24 & 196560 & 11 & $-1, \pm 1 / 2, \pm 1 / 4,0$ & Leech lattice \\
\hline 23 & 4600 & 7 & $-1, \pm 1 / 3,0$ & kissing \\
\hline 22 & 891 & 5 & $-1 / 2,-1 / 8,1 / 4$ & kissing \\
\hline 23 & 552 & 5 & $-1, \pm 1 / 5$ & equiangular lines \\
\hline 22 & 275 & 4 & $-1 / 4,1 / 6$ & kissing \\
\hline 21 & 162 & 3 & $-2 / 7,1 / 7$ & kissing \\
\hline 22 & 100 & 3 & $-4 / 11,1 / 11$ & Higman-Sims \\
\hline$q \frac{q^{3}+1}{q+1}$ & $(q+1)\left(q^{3}+1\right)$ & 3 & $-1 / q, 1 / q^{2}$ & isotropic subspaces \\
\hline & & $q=$ & & ( $q$ a prime power) \\
\hline
\end{tabular}

greatest possible strength given the number of distances occurring in them. (Some but not all sharp configurations are actually $2 m$-designs.)

All sharp arrangements that we know of are listed in Table 1, together with the 600-cell (which is not sharp, but to which our techniques nevertheless apply). The columns list the dimension $n$ of the ambient Euclidean space, the number $N$ of points, the largest $M$ such that the code is a spherical $M$-design, the inner products other than 1 that occur between points in the code, and the name of the code, if any. If $t$ denotes the largest inner product, then each of these codes is the unique $(n, N, t)$ spherical code, except for some of those listed on the last line of the table (the isotropic subspace codes). See Appendix $₫$ for details about uniqueness.

The first six configurations listed in Table 1 are the vertices of certain fulldimensional regular polytopes (specifically, those regular polytopes whose faces are simplices), together with lower-dimensional simplices. The next seven cases are derived from the $E_{8}$ root lattice in $\mathbb{R}^{8}$ and the Leech lattice in $\mathbb{R}^{24}$. The 240-point and 196560-point configurations are the minimal (nonzero) vectors in those lattices. In sphere packing terms, they are the kissing configurations, the points of tangency in the corresponding sphere packings. Each arrangement with the label "kissing" is the kissing configuration of the arrangement above it: each configuration yields a sphere packing in spherical geometry by centering congruent spherical caps at the points, with radius as large as possible without making their interiors overlap. The points of tangency on a given cap form a spherical code in a space of one fewer dimension. In general different spheres in a packing can have different kissing configurations, but that does not occur here because each 
configuration's automorphism group acts transitively on it. (See Chapter 14 of [CS for the details of these configurations.) Some of the kissing configurations are of independent interest. For example, the 27 points on $S^{5}$ form the Schläfli arrangement, which is connected as follows with the classical configuration of 27 lines on a cubic surface. Let $X$ be a smooth cubic surface in $\mathbb{C P}^{3}$. Its divisor class group $\operatorname{Pic}(X)$ is a free abelian group of rank 7 , equipped with a bilinear form of signature $(1,6)$ given by taking intersection numbers (see Proposition 4.8 in $\underline{\mathrm{H}}$, p. 401]). The class $h$ of a hyperplane section has self-intersection number $h^{2}=3$, so its orthogonal complement $h^{\perp}$ is negative definite. Thus, after changing the sign of the metric, $h^{\perp} \otimes_{\mathbb{Z}} \mathbb{R}$ is naturally a Euclidean space. Orthogonally projecting the divisor classes of the 27 lines on $X$ from $\operatorname{Pic}(X) \otimes_{\mathbb{Z}} \mathbb{R}$ to $h^{\perp} \otimes_{\mathbb{Z}} \mathbb{R}$ yields 27 points on a sphere, which form the Schläfli configuration (up to scaling). The sharp configurations with 240,56 , or 16 points have similar interpretations via exceptional curves on del Pezzo surfaces; see Corollary 25.1.1 and Theorem 26.2 in [Man] for details.

The sharp configuration of 552 points in $\mathbb{R}^{23}$ comes from an equiangular arrangement of 276 lines in $\mathbb{R}^{23}$ described by the unique regular two-graph on 276 vertices; see Chapter 11 of GR. It can be derived from the Leech lattice $\Lambda_{24}$ as follows. Choose any $w \in \Lambda_{24}$ with $|w|^{2}=6$. Then the 552 points are the vectors $v \in \Lambda_{24}$ satisfying $|v|^{2}=|w-v|^{2}=4$. These points all lie on a hyperplane, but it does not pass through the origin, so subtract $w / 2$ from each vector to obtain points on a sphere centered at the origin.

The Higman-Sims configuration of 100 points in $\mathbb{R}^{22}$ can be constructed similarly, following Wil]. Choose $w_{1}$ and $w_{2}$ in $\Lambda_{24}$ satisfying $\left|w_{1}\right|^{2}=\left|w_{2}\right|^{2}=6$ and $\left|w_{1}-w_{2}\right|^{2}=4$. Then there are 100 points $v \in \Lambda_{24}$ satisfying $|v|^{2}=\left|w_{1}-v\right|^{2}=$ $\left|w_{2}-v\right|^{2}=4$, and they form the Higman-Sims configuration (on an affine subspace as above).

One might imitate the last two constructions by using the $E_{8}$ lattice instead of the Leech lattice and replacing the norms 6 and 4 with the smallest two norms in $E_{8}$, namely 4 and 2 . That works, but it merely yields the cross polytope in $\mathbb{R}^{7}$ and the simplex in $\mathbb{R}^{6}$.

The last line of the table describes a remarkable family of sharp configurations from CGS. They are the only known sharp configurations not derived from regular polytopes, the $E_{8}$ lattice, or the Leech lattice. The parameter $q$ must be a prime power. When $q=2$, this arrangement is the Schläfli configuration (and it is a spherical 4-design), but for $q>2$ it is different from all the other entries in the table. Points in the configuration correspond to totally isotropic 2-dimensional subspaces of a 4-dimensional Hermitian space over $\mathbb{F}_{q^{2}}$, with the distances between points determined by the dimensions of the intersections of the corresponding subspaces (inner product $-1 / q$ corresponds to intersection dimension 1 ). This construction generalizes to higher-dimensional Hermitian spaces, but the 891-point configuration in $\mathbb{R}^{22}$ appears to be the only other sharp configuration that can be obtained in this way, from totally isotropic 3-dimensional subspaces of a 6 -dimensional Hermitian space over $\mathbb{F}_{4}$.

Antipodal sharp configurations are the same as antipodal tight spherical designs, by Theorem 6.8 in DGS. Much progress has been made towards classifying such designs; see [BMV] for more details. In particular, Table 1 contains all of the antipodal sharp configurations in at most 103 dimensions. 
Table 1 coincides with the list of known cases in which the linear programming bounds for spherical codes are sharp; the list comes from [Lev3, p. 621], except for the 600-cell, which was dealt with in An3 and El. We conjecture that our techniques apply to a configuration if and only if the linear programming bounds for spherical codes prove a sharp bound for it.

In our main theorem, we prove that sharp arrangements and also the 600-cell are global potential energy minima for every completely monotonic potential function:

Theorem 1.2. Let $f:(0,4] \rightarrow \mathbb{R}$ be completely monotonic, and let $\mathcal{C} \subset S^{n-1}$ be a sharp arrangement or the vertices of a regular 600 -cell. If $\mathcal{C}^{\prime} \subset S^{n-1}$ is any subset satisfying $\left|\mathcal{C}^{\prime}\right|=|\mathcal{C}|$, then

$$
\sum_{x, y \in \mathcal{C}^{\prime}, x \neq y} f\left(|x-y|^{2}\right) \geq \sum_{x, y \in \mathcal{C}, x \neq y} f\left(|x-y|^{2}\right) .
$$

If $f$ is strictly completely monotonic, then equality in (1.1) implies that $\mathcal{C}^{\prime}$ is also a sharp configuration or the vertices of a 600-cell (whichever $\mathcal{C}$ is) and the same distances occur in $\mathcal{C}$ and $\mathcal{C}^{\prime}$. In that case, if $\mathcal{C}$ is listed in Table 1 but not on the last line, then $\mathcal{C}^{\prime}=A \mathcal{C}$ for some $A \in O(n)$ (i.e., $\mathcal{C}^{\prime}$ and $\mathcal{C}$ are isometric).

Uniqueness does not necessarily hold if the hypothesis that $f$ must be strictly completely monotonic is removed (consider a constant potential function). Furthermore, the configurations from the last line in Table 1 are not always unique. The construction in CGS builds such a configuration given any generalized quadrangle with parameters $\left(q, q^{2}\right)$. For $q \leq 3$ such quadrangles are unique (see Section 9 in [CGS]), as are the spherical codes derived from them, but for larger $q$ that is not always true. Whenever distinct generalized quadrangles exist, the construction yields distinct spherical codes. In particular, as explained in CGS, one can construct such quadrangles from ovoids in $\mathbb{F}_{q} \mathbb{P}^{3}$. When $q=2^{k}$ with $k$ odd and $k>1$, there are at least two distinct ovoids, namely the elliptic quadric ovoid and the Suzuki-Tits ovoid (see [T]). They yield distinct generalized quadrangles and thus distinct universally optimal spherical codes.

To illustrate the techniques used to prove Theorem 1.2, in this section we will prove the simplest possible case, namely when $|\mathcal{C}| \leq n+1$ (in which case $\mathcal{C}$ is a simplex). The full proof is in Section 6

Proof of special case. Let $N=|\mathcal{C}|$, and suppose $N \leq n+1$. Then $\mathcal{C}$ is a regular simplex with inner product $-1 /(N-1)$ between distinct points, so the squared Euclidean distance between distinct points is $2+2 /(N-1)$ (because $|x-y|^{2}=$ $2-2\langle x, y\rangle$ when $\left.|x|^{2}=|y|^{2}=1\right)$. For this special case, we only require $f$ to be decreasing and convex, rather than completely monotonic.

Let

$$
h(x)=f(2+2 /(N-1))+f^{\prime}(2+2 /(N-1))(x-(2+2 /(N-1))),
$$

so $h$ is the tangent line to $f$ at $2+2 /(N-1)$. Because $f$ is convex, $f(x) \geq h(x)$ for all $x \in(0,4]$, and if $f$ is strictly convex, then equality holds if and only if $x=2+2 /(N-1)$.

Suppose $\mathcal{C}^{\prime}$ is any subset of $S^{n-1}$ with $\left|\mathcal{C}^{\prime}\right|=N$. Then

$$
\sum_{x, y \in \mathcal{C}^{\prime}, x \neq y} f\left(|x-y|^{2}\right) \geq \sum_{x, y \in \mathcal{C}^{\prime}, x \neq y} h\left(|x-y|^{2}\right),
$$


and the right side equals

$$
\begin{array}{r}
N(N-1) f(2+2 /(N-1)) \\
-(2+2 /(N-1)) N(N-1) f^{\prime}(2+2 /(N-1)) \\
+f^{\prime}(2+2 /(N-1)) \sum_{x, y \in \mathcal{C}^{\prime}}|x-y|^{2} .
\end{array}
$$

We have

$$
\begin{aligned}
\sum_{x, y \in \mathcal{C}^{\prime}}|x-y|^{2} & =\sum_{x, y \in \mathcal{C}^{\prime}}(2-2\langle x, y\rangle) \\
& =2 N^{2}-2\left|\sum_{x \in \mathcal{C}^{\prime}} x\right|^{2} \\
& \leq 2 N^{2} .
\end{aligned}
$$

Because $f^{\prime}(2+2 /(N-1)) \leq 0$, we find that the potential energy of $\mathcal{C}^{\prime}$ is at least

$$
\begin{array}{r}
N(N-1) f(2+2 /(N-1)) \\
-(2+2 /(N-1)) N(N-1) f^{\prime}(2+2 /(N-1)) \\
+2 N^{2} f^{\prime}(2+2 /(N-1)),
\end{array}
$$

which equals

$$
N(N-1) f(2+2 /(N-1)) \text {. }
$$

That is the potential energy of $\mathcal{C}$, so we have proved that

$$
\sum_{x, y \in \mathcal{C}^{\prime}, x \neq y} f\left(|x-y|^{2}\right) \geq \sum_{x, y \in \mathcal{C}, x \neq y} f\left(|x-y|^{2}\right) .
$$

When $f$ is strictly convex, equality holds only if $|x-y|^{2}=2+2 /(N-1)$ for all $x, y \in \mathcal{C}^{\prime}$ with $x \neq y$, in which case $\mathcal{C}^{\prime}$ is a regular simplex.

We use the term universal optimality to describe the conclusion of Theorem 1.2 ;

Definition 1.3. A finite subset $\mathcal{C} \subset S^{n-1}$ is universally optimal if it (weakly) minimizes potential energy among all configurations of $|\mathcal{C}|$ points on $S^{n-1}$ for each completely monotonic potential function.

As the name suggests, every universally optimal spherical code is indeed an optimal spherical code, in the sense of maximizing the minimal distance between points. For the potential function $f(r)=1 / r^{s}$, the leading term in the large- $s$ asymptotics of the potential energy comes from the minimal distance. If there were a spherical code with the same number of points but a larger minimal distance, then it would have lower potential energy when $s$ is sufficiently large.

As we mentioned above, the optimality of sharp configurations as spherical codes is not new. Levenshtein proved in Lev2 that every sharp configuration is a maximal spherical code, by using linear programming bounds. For the $E_{8}$ and Leech minimal vectors, this amounts to the solution of the kissing problem by Levenshtein and independently by Odlyzko and Sloane in Lev1, OS]; see [Lev3] for an overview of the other cases, except for the 600-cell, for which optimality follows from the bound in [Bö] (see also [An3] and [El] for a solution using linear programming bounds). Our theorem can thus be viewed as a continuum of optimality results connecting the kissing problem and Levenshtein's theorem to the results of [KY1] and [An2]. 
Certain other known optimality results can also be derived. For example, KY1 and An2 prove that the $E_{8}$ and Leech minimal vectors maximize the sum of the distances between pairs of points. That is the case $f(r)=2-r^{1 / 2}$ in Theorem 1.2. Similarly, taking $f(r)=\log (4 / r)$ shows that sharp configurations maximize the product of the distances between pairs of distinct points.

When testing whether a configuration is universally optimal, it is helpful to be able to restrict to a smaller class of potential functions. Theorem 9b in Wid, p. 154] implies that on each compact subinterval of $(0,4]$, every completely monotonic function on $(0,4]$ can be approximated uniformly by nonnegative linear combinations of the functions $r \mapsto(4-r)^{k}$ with $k \in\{0,1,2, \ldots\}$. For example,

$$
\frac{1}{r^{s}}=\sum_{k \geq 0}\left(\begin{array}{c}
s+k-1 \\
k
\end{array}\right) \frac{(4-r)^{k}}{4^{k+s}} .
$$

Thus, a configuration is universally optimal if and only if it is optimal for each of the potential functions $f(r)=(4-r)^{k}$.

The conclusion of optimality under all completely monotonic potential functions is quite strong, and computer experiments suggest that it is rarely true in other cases. For example, it provably fails in the case of five points on $S^{2}$. Schütte and van der Waerden proved in $\mathrm{SchW}$ that the minimal angle in such a code cannot be greater than $\pi / 2$. Their analysis shows that all optimal codes consist of a pair of antipodal points with three points on the orthogonal plane. Among such codes, the only one that could be universally optimal is the one in which the three points form an equilateral triangle. However, when $f(r)=(4-r)^{7}$, there is a configuration with lower energy: take a single point together with four points equidistant from it and forming a square in the opposite hemisphere (the size of the square is chosen so as to minimize energy). Thus, the only candidate for a five-point universal optimum is not universally optimal. Furthermore, it is not even a local minimum for energy when $f(r)=(4-r)^{k}$ with $k$ sufficiently large or $f(r)=1 / r^{s}$ with $s$ sufficiently large.

Leech proved a far stronger theorem in Lee. He classified the configurations on $S^{2}$ that are in equilibrium under all force laws, which is a necessary but not sufficient condition for universal optimality. His theorem implies that there are no universal optima in $\mathbb{R}^{3}$ beyond those in Table 1 by ruling out all cases except for five points (see [Lee, p. 89]). We conjecture that for each $n \geq 3$ there are only finitely many universal optima in $\mathbb{R}^{n}$, but we cannot prove it even for $n=4$.

We do not know of any other spherical codes that could be added to Table1, but some may well exist. One surprising case in which universal optimality fails is the vertices of the regular 24-cell on $S^{3}$ (equivalently, the $D_{4}$ root system). It is not a sharp configuration, because it has four inner products besides 1 while it is only a spherical 5 -design, but one might still hope that it would be universally optimal. It appears to be optimal for many inverse power law potentials, but even this weaker conclusion is surprisingly subtle: for example, when one simulates 24 randomly chosen points on $S^{3}$ under the potential function $f(r)=1 / r$, more than $90 \%$ of the time one reaches a local optimum with potential energy 334.096 ... (an algebraic number of degree 20), which is only slightly worse than the potential energy 334 of the 24-cell. In other words, there is a suboptimal arrangement with a much larger basin of attraction. This arrangement belongs to an explicit one-parameter family of configurations analyzed in [CCEK]. In that paper, some of them were shown 
to improve on the 24-cell for certain completely monotonic potential functions, including $f(r)=(4-r)^{k}$ with $8 \leq k \leq 13$ and $f(r)=e^{-3 r}$. The 24-cell is therefore not universally optimal.

The remaining regular polytopes (the cubes in $\mathbb{R}^{n}$ for $n \geq 3$, the dodecahedron in $\mathbb{R}^{3}$, and the 120 -cell in $\mathbb{R}^{4}$ ) are also not universally optimal, for a less interesting reason: they are not even optimal spherical codes. Antiprisms improve on cubes, and [S] deals with the other two cases. Thus, a full-dimensional regular polytope is universally optimal if and only if all its faces are simplices.

One might also wonder about the $(4,10,1 / 6)$ code that is the kissing configuration of the universally optimal $(5,16,1 / 5)$ code. It consists of the midpoints of the edges of a regular simplex. This code is not universally optimal, because for the potential functions $f(r)=(4-r)^{k}$ with $3 \leq k \leq 6$, arranging two regular pentagons on orthogonal planes yields lower potential energy. For $k \leq 2$ their potential energies agree; for $k \geq 7$, the $(4,10,1 / 6)$ code is superior to the two pentagons, but we do not know whether it is optimal in these cases. Note that the kissing configuration of this $(4,10,1 / 6)$ code is a $(3,6,1 / 7)$ code that is not even optimal, let alone universally optimal.

The $(4,10,1 / 6)$ code described above is a spherical 2-design in which only two inner products occur between distinct points. It follows that replacing the bound $2 m-1$ with $2 m-2$ in Definition 1.1 would make Theorem 1.2 false.

Probably the $(21,336,1 / 5)$ kissing configuration of the $(22,891,1 / 4)$ code is not universally optimal, but in this case we do not know a better configuration. We also do not know whether universal optimality holds for the $(20,105,1 / 8)$ kissing configuration of the $(21,162,1 / 7)$ code or the $(21,77,1 / 12)$ kissing configuration of the $(22,100,1 / 11)$ code. These configurations are not sharp, so Theorem 1.2 does not apply.

We do not know whether there exist universally optimal spherical codes whose universal optimality cannot be proved by our methods. It seems likely that such codes exist, but we have no candidates to propose.

Another important question not addressed by our methods is whether there are other local minima for potential energy in the cases listed in Table 1, In certain special cases the existence of other minima is trivial; for example, for $f(r)=4-r$, a configuration minimizes potential energy if and only if its centroid is the origin, so there is a positive-dimensional space of global minima (when $N>3$ ). In general, that is true for $f(r)=(4-r)^{k}$ if $k$ is small relative to the number of points in the configuration. (It was proved in [SZ that spherical $k$-designs exist on $S^{n-1}$ for all sufficiently large numbers of points. Spherical $k$-designs automatically minimize energy for $f(r)=(4-r)^{k}$, and because the set of such designs is closed under rotation and taking unions they exist in positive-dimensional families once the number of points is large enough.) For sufficiently large $k$ or for inverse power laws, the set of local minima appears to be finite, but sometimes there are several. When the number of points is large, there can be vast numbers of local minima. For example, experiments suggest that for 120 points on $S^{3}$ under the harmonic power law potential, there are more than five thousand distinct local minima, and perhaps far more than that.

It is natural to ask whether the requirement of complete monotonicity could be weakened. For example, regular simplices are optimal for all decreasing, convex potential functions, although not for all decreasing potential functions. That does 
not always hold. Specifically, the icosahedron is not even a local minimum for the decreasing, convex potential function $f(r)=r^{3}-48 r+128$, but rather a saddle point. One can show using Proposition 4.1 that the global minimum for twelve points in $\mathbb{R}^{3}$ is obtained degenerately by letting them coincide in triples at the vertices of a tetrahedron. More generally, repeating the vertices of a regular simplex in $\mathbb{R}^{n}$ is optimal whenever the number of points is a multiple of $n+1$. This potential function therefore behaves quite differently from completely monotonic potential functions.

The potential function $f(r)=r^{3}-48 r+128$ from the previous paragraph is convex, but $r \mapsto f\left(r^{2}\right)$ is not (so the potential energy is not convex as a function of distance, as opposed to distance squared). At the cost of slightly greater complexity one can obtain that stronger property: if $f(r)=r^{7}-7 \cdot 2^{14} \cdot \sqrt{r}+13 \cdot 2^{14}$, then $r \mapsto f\left(r^{2}\right)$ is decreasing and convex, as is $f$, but the icosahedron is not a local minimum for potential energy.

Although it is not enough to assume that the potential function is decreasing and convex, in each case only finitely many derivative conditions are needed. For each sharp configuration, our methods produce an explicit bound $K$ such that optimality holds for a potential function $f \in C^{K}((0,4])$ whenever $(-1)^{k} f^{(k)}(r) \geq 0$ for all $r \in(0,4]$ and $k \leq K$. We will not discuss this issue further, but it is worth recording the result: for a sharp configuration with $m$ inner products between distinct points, we can take $K=2 m$; furthermore, if $(-1)^{k} f^{(k)}(r)>0$ for all $r \in(0,4)$ and $k \leq 2 m$, then every configuration of the same size and dimension with this potential energy is sharp (and the same distances occur between points in it as in the original configuration). For antipodal configurations a more refined construction can be used to reduce both of these bounds by 1 , but it is implausible that they could be substantially reduced using our methods.

We conclude the introduction with one negative result in arbitrary dimensions, namely that there are no universal optima in between the simplex and cross polytope:

Proposition 1.4. If $n+1<N<2 n$, then there is no $N$-point universally optimal configuration on $S^{n-1}$.

Proof. An optimal code of this size has the following structure (see, for example, Theorem 6.2.1 in [BöJr] and the remark following it). There are $N-n$ pairwise orthogonal subspaces of $\mathbb{R}^{n}$ whose span is $\mathbb{R}^{n}$, each containing $d+1$ points of the code if its dimension is $d$. If the code is universally optimal, then each of these subspaces must contain a regular simplex: because of orthogonality, distances between points in different subspaces are constant, so within each subspace we must have a universally optimal code. For the same reason, every union of some of the component simplices must be universally optimal as well. Thus, without loss of generality we can assume that $N=n+2$. We can also assume that $n \geq 4$ because the case of $n=3$ and $N=5$ was dealt with earlier in this section.

Suppose the two regular simplices have $i+1$ and $n-i+1$ points. For the potential function $f(r)=(4-r)^{k}$, the energy of the configuration equals

$$
i(i+1)\left(2-\frac{2}{i}\right)^{k}+(n-i)(n-i+1)\left(2-\frac{2}{n-i}\right)^{k}+(i+1)(n-i+1) 2^{k+1} .
$$

For $k=2$ that equals $4 n(n+1)-4 n /(i(n-i))$, from which it follows that the potential energy is minimized exactly when $i=\lfloor n / 2\rfloor$ or $i=\lceil n / 2\rceil$. As $k \rightarrow \infty$, 
the energy is asymptotic to $(i+1)(n-i+1) 2^{k+1}$, which is minimized exactly when $i=1$ or $i=n-1$ (and hence not at $i=\lfloor n / 2\rfloor$ or $i=\lceil n / 2\rceil$, because $n \geq 4$ ). Thus, universal optimality fails, as asserted.

\section{BACKGROUND}

In this section we briefly review the tools required for the proof of Theorem 1.2 .

2.1. Hermite interpolation. Recall that Hermite interpolation is a generalization of Lagrange interpolation in which one computes a polynomial that matches not only the values but also some derivatives of a function at some specified points. More precisely, suppose we are given $f \in C^{\infty}([a, b])$, distinct points $t_{1}, \ldots, t_{m} \in$ $[a, b]$, and positive integers $k_{1}, \ldots, k_{m}$. We wish to find a polynomial of degree less than $D=k_{1}+k_{2}+\cdots+k_{m}$ such that for $1 \leq i \leq m$ and $0 \leq k<k_{i}$,

$$
p^{(k)}\left(t_{i}\right)=f^{(k)}\left(t_{i}\right) \text {. }
$$

In other words, $p$ and $f$ agree to order $k_{i}$ at $t_{i}$. A unique such polynomial always exists, because the linear map that takes a polynomial $p$ of degree less than $D$ to

$$
\left(p\left(t_{1}\right), p^{\prime}\left(t_{1}\right), \ldots, p^{\left(k_{1}-1\right)}\left(t_{1}\right), p\left(t_{2}\right), p^{\prime}\left(t_{2}\right), \ldots, p^{\left(k_{m}-1\right)}\left(t_{m}\right)\right)
$$

is injective (and hence surjective as well).

One fundamental fact about Hermite interpolation is the following remainder formula (see Theorem 3.5.1 in [D, p. 68]):

Lemma 2.1. Under the hypotheses above, for each $t \in[a, b]$ there exists $\xi \in(a, b)$ such that $\min \left(t, t_{1}, \ldots, t_{m}\right)<\xi<\max \left(t, t_{1}, \ldots, t_{m}\right)$ and

$$
f(t)-p(t)=\frac{f^{(D)}(\xi)}{D !}\left(t-t_{1}\right)^{k_{1}} \ldots\left(t-t_{m}\right)^{k_{m}} .
$$

Because the standard proof is short, we reproduce it here for the convenience of the reader:

Proof. Let

$$
g(t)=\frac{f(t)-p(t)}{\left(t-t_{1}\right)^{k_{1}} \ldots\left(t-t_{m}\right)^{k_{m}}} .
$$

For fixed $t \in[a, b] \backslash\left\{t_{1}, \ldots, t_{m}\right\}$ (the lemma is trivial for $t \in\left\{t_{1}, \ldots, t_{m}\right\}$ ), consider the function

$$
s \mapsto f(s)-p(s)-g(t)\left(s-t_{1}\right)^{k_{1}} \ldots\left(s-t_{m}\right)^{k_{m}} .
$$

By construction, for each $i$ it vanishes at $t_{i}$ to order $k_{i}$, and it also vanishes at $t$, so it has $D+1$ roots in the interval

$$
\left[\min \left(t, t_{1}, \ldots, t_{m}\right), \max \left(t, t_{1}, \ldots, t_{m}\right)\right] .
$$

By iterated use of Rolle's theorem, there exists $\xi$ in the interior of this interval at which the $D$-th derivative vanishes. At that point,

$$
f^{(D)}(\xi)-g(t) D !=0
$$

which gives the desired formula for $g(t)$. 
This formula will play a fundamental role later in the paper, but we will also need a stronger result. Recall that a function is absolutely monotonic on an interval if it is $C^{\infty}$ and it and all its derivatives are nonnegative on that interval (see Wid, p. 144]). Unfortunately, the terms "completely monotonic" and "absolutely monotonic" are easy to confuse, but they are standard terms. As a mnemonic, associate "absolutely monotonic" with "absolute value" to remember which one has no signs. We call a function strictly absolutely monotonic on an interval if it and all its derivatives are nonnegative on the interval and strictly positive on its interior.

Proposition 2.2. Under the hypotheses above, suppose further that $f$ is absolutely monotonic on $(a, b)$. Then

$$
\frac{f(t)-p(t)}{\left(t-t_{1}\right)^{k_{1}} \ldots\left(t-t_{m}\right)^{k_{m}}}
$$

is also absolutely monotonic on $(a, b)$. If $f$ is strictly absolutely monotonic, then

$$
\frac{f(t)-p(t)}{\left(t-t_{1}\right)^{k_{1}} \ldots\left(t-t_{m}\right)^{k_{m}}}
$$

is as well.

Of course, when we write quotients such as this one, we define them by continuity when $t=t_{i}$ for some $i$ so that they become $C^{\infty}$ functions. It seems plausible that Proposition 2.2 is known, but we do not know where to find it in the literature. The hypothesis that $t_{1}, \ldots, t_{m}$ lie in the interval $[a, b]$ is crucial (it is part of the setup above, but it is worth reiterating it here).

Before proving Proposition 2.2, it is convenient to introduce systematic notation for Hermite interpolations. Given a polynomial $g$ with $\operatorname{deg}(g) \geq 1$, let $H(f, g)$ denote the polynomial of degree less than $\operatorname{deg}(g)$ that agrees with $f$ at each root of $g$ to the order of that root. (If $f$ is a polynomial, then $H(f, g)$ is its remainder modulo $g$ in polynomial division.) In other words, if $g(t)$ vanishes to order $k$ at $t=s$, then

$$
f(t)-H(f, g)(t)=O\left((t-s)^{k}\right)
$$

as $t \rightarrow s$. It follows that the function $Q(f, g)$ defined by

$$
Q(f, g)(t)=\frac{f(t)-H(f, g)(t)}{g(t)}
$$

extends to a $C^{\infty}$ function at the roots of $g$. Proposition 2.2 is the assertion that if $g(t)=\left(t-t_{1}\right)^{k_{1}} \ldots\left(t-t_{m}\right)^{k_{m}}$, then $Q(f, g)$ is absolutely monotonic.

Proof. The proof rests on two key properties. The first is that

$$
Q\left(f, g_{1} g_{2}\right)=Q\left(Q\left(f, g_{1}\right), g_{2}\right),
$$

which follows from the uniqueness of the Hermite interpolation:

$$
Q\left(Q\left(f, g_{1}\right), g_{2}\right)=\frac{f-H\left(f, g_{1}\right)-g_{1} H\left(Q\left(f, g_{1}\right), g_{2}\right)}{g_{1} g_{2}},
$$

but $H\left(f, g_{1} g_{2}\right)$ is the unique polynomial of degree less than $\operatorname{deg}\left(g_{1}\right)+\operatorname{deg}\left(g_{2}\right)$ such that $\left(f-H\left(f, g_{1} g_{2}\right)\right) /\left(g_{1} g_{2}\right)$ extends to a $C^{\infty}$ function everywhere. Thus, $H\left(f, g_{1} g_{2}\right)=H\left(f, g_{1}\right)+g_{1} H\left(Q\left(f, g_{1}\right), g_{2}\right)$ and $Q\left(f, g_{1} g_{2}\right)=Q\left(Q\left(f, g_{1}\right), g_{2}\right)$.

The second property is that if $g_{0}(t)=\left(t-s_{0}\right)^{n}$, then

$$
Q\left(f, g_{0}\right)\left(s_{0}\right)=\frac{f^{(n)}\left(s_{0}\right)}{n !} .
$$


This follows immediately from the Taylor expansion.

We must show that for $n \geq 0$ and $t \in(a, b)$,

$$
Q(f, g)^{(n)}(t) \geq 0 .
$$

When $n=0$, that follows from Lemma 2.1, which asserts the existence of $\xi \in(a, b)$ (depending on $t$ ) such that

$$
Q(f, g)(t)=\frac{f^{(\operatorname{deg}(g))}(\xi)}{\operatorname{deg}(g) !} \geq 0 .
$$

For larger $n$, given $s_{0} \in(a, b)$, define $g_{0}(t)=\left(t-s_{0}\right)^{n}$. Then

$$
\frac{Q(f, g)^{(n)}\left(s_{0}\right)}{n !}=Q\left(Q(f, g), g_{0}\right)\left(s_{0}\right)=Q\left(f, g g_{0}\right)\left(s_{0}\right) \geq 0 .
$$

This completes the proof when $f$ is absolutely monotonic. The case of strictly absolutely monotonic functions works exactly the same way.

It follows immediately from continuity that the statement of Proposition 2.2 also holds when the open interval $(a, b)$ is replaced with a half-open or closed interval.

2.2. Positive-definite kernels. A continuous function $K: S^{n-1} \times S^{n-1} \rightarrow \mathbb{R}$ is a positive-definite kernel if for all $k$ and all $x_{1}, \ldots, x_{k} \in S^{n-1}$, the $k \times k$ matrix whose $i, j$ entry is $K\left(x_{i}, x_{j}\right)$ is positive semidefinite. In other words, for all $t_{1}, \ldots, t_{k} \in \mathbb{R}$,

$$
\sum_{1 \leq i, j \leq k} t_{i} t_{j} K\left(x_{i}, x_{j}\right) \geq 0
$$

The most important special case is when $t_{1}=\cdots=t_{k}=1$ : for every finite subset $\mathcal{C} \subset S^{n-1}$

$$
\sum_{x, y \in \mathcal{C}} K(x, y) \geq 0
$$

Positive-definite kernels $K$ such that $K(x, y)$ depends only on the distance $|x-y|$ between $x$ and $y$ will be particularly important. There is a simple representationtheoretic construction of such kernels, which Schoenberg proved gives all of them (see $[\mathrm{Sch}]$ ). As a unitary representation of $O(n)$, the Hilbert space $L^{2}\left(S^{n-1}\right)$ breaks up as a completed orthogonal direct sum of infinitely many finite-dimensional representations:

$$
L^{2}\left(S^{n-1}\right)=\widehat{\bigoplus_{\ell \geq 0}} V_{\ell}
$$

where $V_{\ell}$ is the space of spherical harmonics of degree $\ell$ (i.e., restrictions to $S^{n-1}$ of homogeneous polynomials on $\mathbb{R}^{n}$ of degree $\ell$ that are in the kernel of the Euclidean Laplacian). Note that spherical harmonics of different degrees are orthogonal, which will play a useful role later. It is also important to know that every polynomial on $\mathbb{R}^{n}$ has the same restriction to $S^{n-1}$ as some unique linear combination of spherical harmonics. For more details on spherical harmonics, see $\S 2$ of Chapter IV in [StW].

For each $\ell \geq 0$ and each $x \in S^{n-1}$, there is a unique reproducing kernel ev $_{\ell, x} \in V_{\ell}$, defined by requiring that for all $f \in V_{\ell}$,

$$
\left\langle f, \mathrm{ev}_{\ell, x}\right\rangle=f(x) .
$$


(We use Hermitian forms that are conjugate-linear in the second variable.) To construct ev $_{\ell, x}$ explicitly, choose an orthonormal basis $e_{\ell, 1}, \ldots, e_{\ell, \operatorname{dim} V_{\ell}}$ of $V_{\ell}$ and define

$$
\operatorname{ev}_{\ell, x}(y)=\overline{e_{\ell, 1}(x)} e_{\ell, 1}(y)+\cdots+\overline{e_{\ell, \operatorname{dim} V_{\ell}(x)}} e_{\ell, \operatorname{dim} V_{\ell}}(y) .
$$

Define the function $K_{\ell}: S^{n-1} \times S^{n-1} \rightarrow \mathbb{C}$ by

$$
K_{\ell}(x, y)=\left\langle\mathrm{ev}_{\ell, x}, \mathrm{ev}_{\ell, y}\right\rangle=\mathrm{ev}_{\ell, x}(y) .
$$

It is straightforward to check that $\mathrm{ev}_{\ell, A x}=\mathrm{ev}_{\ell, x} \circ A^{-1}$ for $A \in O(n)$. Because $O(n)$ acts distance-transitively on $S^{n-1}$ (i.e., if $|u-v|=|x-y|$, then there exists $A \in O(n)$ such that $A u=x$ and $A v=y), K_{\ell}(x, y)$ depends only on the distance between $x$ and $y$. That implies that it is real-valued, since

$$
K_{\ell}(x, y)=\overline{K_{\ell}(y, x)}
$$

follows immediately from the definition while $K_{\ell}(x, y)=K_{\ell}(y, x)$ since $|x-y|=$ $|y-x|$.

The key property of $K_{\ell}$ is that it is a positive-definite kernel:

$$
\sum_{1 \leq i, j \leq k} t_{i} t_{j} K_{\ell}\left(x_{i}, x_{j}\right)=\left|\sum_{1 \leq i \leq k} t_{i} \operatorname{ev}_{\ell, x_{i}}\right|^{2} \geq 0 .
$$

All convergent nonnegative infinite linear combinations of these functions are still positive-definite kernels, and Schoenberg proved that those are the only continuous positive-definite kernels that depend only on the distance between points.

One can compute $K_{\ell}$ explicitly. Because $K_{\ell}(x, y)$ depends only on the distance between $x$ and $y$, we can write $K_{\ell}(x, y)=C_{\ell}(\langle x, y\rangle)$ for some function $C_{\ell}$. To compute $C_{\ell}$, we use the orthogonality between spherical harmonics of different degrees. If we fix a point on the sphere and project orthogonally onto the line through the origin and that point, then the surface measure on the sphere projects to a constant times the measure

$$
\left(1-t^{2}\right)^{(n-3) / 2} d t
$$

on the interval $[-1,1]$, and the orthogonality property amounts to saying that $C_{0}, C_{1}, \ldots$ are orthogonal polynomials with respect to that measure, with $C_{i}$ having degree $i$. That determines these functions up to multiplication by a positive scalar (positive because $C_{i}(1)>0$ ), and pinning down that factor is irrelevant for our purposes.

These orthogonal polynomials are known as the ultraspherical (or Gegenbauer) polynomials. The standard notation is $C_{i}^{\lambda}(t)$ for the degree $i$ polynomial orthogonal with respect to $\left(1-t^{2}\right)^{\lambda-1 / 2} d t$ on $[-1,1]$ (for $\left.S^{n-1}, \lambda=n / 2-1\right)$. These polynomials are normalized so that $C_{0}^{\lambda}(t)=1$ and $C_{1}^{\lambda}(t)=2 \lambda t$, and they satisfy the recurrence

$$
i C_{i}^{\lambda}(t)=2(i+\lambda-1) t C_{i-1}^{\lambda}(t)-(i+2 \lambda-2) C_{i-2}^{\lambda}(t)
$$

for $i \geq 2$. See [AAR, p. 303] for more details on ultraspherical polynomials, as well as Chapter 9 of [AAR for spherical harmonics. We always assume $\lambda \geq 0$ (equivalently, $n \geq 2$ ), because the one-dimensional case is trivial and its ultraspherical polynomials are poorly normalized: above degree 0 they have negative leading coefficients, and $C_{1}^{-1 / 2}$ is not a positive-definite kernel.

Whenever we refer to the ultraspherical coefficients of a function, we mean its coefficients in terms of ultraspherical polynomials (where the parameter $\lambda$ is implicit 
in the context). A positive-definite function will be a function whose ultraspherical coefficients are nonnegative.

One fundamental fact about positive-definite functions is that they are closed under taking products. Equivalently, the product of two ultraspherical polynomials is a nonnegative linear combination of ultraspherical polynomials (for $\lambda \geq 0$ ). By orthogonality, proving that amounts to showing that

$$
\int_{-1}^{1} C_{i}(t) C_{j}(t) C_{k}(t)\left(1-t^{2}\right)^{(n-3) / 2} d t \geq 0
$$

for all $i, j, k \geq 0$. Let $\mu$ denote the surface measure on $S^{n-1}$. Then using the expansion

$$
K_{\ell}(x, y)=\sum_{m=1}^{\operatorname{dim} V_{\ell}} \overline{e_{\ell, m}(x)} e_{\ell, m}(y)
$$

in terms of a choice of orthonormal basis $e_{\ell, 1}, \ldots, e_{\ell, \operatorname{dim} V_{\ell}}$ for each $V_{\ell}$ shows that (2.1) holds if and only if

$$
\sum_{a=1}^{\operatorname{dim} V_{i}} \sum_{b=1}^{\operatorname{dim}} \sum_{c=1}^{V_{j}} \int e_{i, a}(x) e_{j, b}(x) e_{k, c}(x) d \mu(x) \overline{e_{i, a}(y)} \overline{e_{j, b}(y)} \overline{e_{k, c}(y)} \geq 0
$$

for some (equivalently, all) $y \in S^{n-1}$. Integrating over $y$ yields

$$
\sum_{a=1}^{\operatorname{dim} V_{i}} \sum_{b=1}^{\operatorname{dim} V_{j}} \sum_{c=1}^{\operatorname{dim} V_{k}}\left|\int e_{i, a}(x) e_{j, b}(x) e_{k, c}(x) d \mu(x)\right|^{2},
$$

which is visibly nonnegative.

This result also follows from the Schur product theorem: the set of positive semidefinite matrices of a given size is closed under taking Hadamard products (i.e., taking the product entry by entry). See Theorem 7.5.3 in [HJ, p. 458]. That approach leads to the proof of Schoenberg's characterization of positive-definite kernels. Suppose $(x, y) \mapsto f(\langle x, y\rangle)$ is a positive-definite kernel. By the Schur product theorem, the same is true for $(x, y) \mapsto f(\langle x, y\rangle) C_{i}(\langle x, y\rangle)$ for each $i$. It follows that the integral of this function over $(x, y) \in S^{n-1} \times S^{n-1}$ is nonnegative, and hence for fixed $y$ the integral over $x \in S^{n-1}$ is nonnegative (because it is independent of $y$ ). In other words, the $i$-th ultraspherical coefficient of $f$ must be nonnegative.

We call a polynomial strictly positive definite if all its ultraspherical coefficients are strictly positive (up to its degree, of course). The product of strictly positivedefinite polynomials is strictly positive definite; the proof rests on the fact that in the expansion of $C_{i}^{\lambda} C_{j}^{\lambda}$, the coefficient of $C_{i+j}^{\lambda}$ is positive (because all these polynomials have positive leading coefficients).

One useful application of ultraspherical polynomials is the following simple test for spherical design strength (Theorem 5.5 in [DGS]): a configuration $\mathcal{C} \subset S^{n-1}$ is a spherical $M$-design if and only if

$$
\sum_{x, y \in \mathcal{C}} C_{i}^{n / 2-1}(\langle x, y\rangle)=0
$$

for $1 \leq i \leq M$. That property depends only on the distance distribution of $\mathcal{C}$ (i.e, which distances occur between points in $\mathcal{C}$ and the multiplicities of those distances). 
To prove this characterization of designs, observe that the identity

$$
\sum_{x, y \in \mathcal{C}} C_{i}^{n / 2-1}(\langle x, y\rangle)=\sum_{x, y \in \mathcal{C}}\left\langle\mathrm{ev}_{i, x}, \mathrm{ev}_{i, y}\right\rangle=\left|\sum_{x \in \mathcal{C}} \mathrm{ev}_{i, x}\right|^{2}
$$

shows that (2.2) holds if and only if all of the degree $i$ spherical harmonics sum to zero over $\mathcal{C}$.

\section{ORthogonal POLYNOMials}

The proof of Theorem 1.2 involves expansions as nonnegative linear combinations of orthogonal polynomials. In this section we prove a theorem about such expansions that will play a fundamental role later in the paper. We prove it in greater generality than is required for Theorem 1.2, because it is no more difficult and the proof is of independent interest.

Let $\mu$ be any Borel measure on $\mathbb{R}$ such that all polynomials are integrable with respect to $\mu$ and for all polynomials $p$,

$$
\int p(t)^{2} d \mu(t)>0
$$

if $p$ is not identically zero. Equivalently, for all polynomials $p$ such that $p(t) \geq 0$ for all $t$ but $p$ is not identically zero,

$$
\int p(t) d \mu(t)>0
$$

(Every such polynomial is a sum of two squares of polynomials; see for example Problem 44 in Part VI of [PS] and its solution.)

Let $p_{0}, p_{1}, \ldots$ be the monic orthogonal polynomials for $\mu$, with $\operatorname{deg}\left(p_{i}\right)=i$. In other words, for $i \neq j$

$$
\int p_{i}(t) p_{j}(t) d \mu(t)=0
$$

It is known that for each $\alpha \in \mathbb{R}$, the polynomial $p_{n}+\alpha p_{n-1}$ has $n$ distinct real roots, which are interlaced with the roots of $p_{n-1}$ (see Theorem 3.3.4 in [Sz]).

Theorem 3.1. Let $\alpha$ be any real number, and let

$$
r_{1}<r_{2}<\cdots<r_{n}
$$

be the roots of $p_{n}+\alpha p_{n-1}$. Then for $k<n$,

$$
\prod_{i=1}^{k}\left(t-r_{i}\right)
$$

has positive coefficients in terms of $p_{0}(t), p_{1}(t), \ldots, p_{k}(t)$.

The proof is conceptually simple, but it involves some technicalities. We begin with the simplest case, namely $k=n-1$ :

Proposition 3.2. Let $\alpha$ be any real number, and let $r$ be the largest root of $p_{n}+$ $\alpha p_{n-1}$. Then

$$
\frac{p_{n}(t)+\alpha p_{n-1}(t)}{t-r}
$$

has positive coefficients in terms of $p_{0}(t), p_{1}(t), \ldots, p_{n-1}(t)$. 
Proposition 3.2 follows from the Christoffel-Darboux formula (Theorem 5.2.4 in (AAR), but we prove it directly to illustrate the technique we will apply to the general case.

Proof. Define $c_{0}, \ldots, c_{n-1}$ so that

$$
\frac{p_{n}(t)+\alpha p_{n-1}(t)}{t-r}=\sum_{\ell=0}^{n-1} c_{\ell} p_{\ell}(t) .
$$

For $\ell \leq n-1$, it follows from orthogonality that

$$
\int\left(p_{n}(t)+\alpha p_{n-1}(t)\right) \frac{p_{\ell}(t)-p_{\ell}(r)}{t-r} d \mu(t)=0,
$$

because

$$
\frac{p_{\ell}(t)-p_{\ell}(r)}{t-r}
$$

is a polynomial of degree $\ell-1$ and hence orthogonal to both $p_{n}(t)$ and $p_{n-1}(t)$. Thus,

$$
\int \frac{p_{n}(t)+\alpha p_{n-1}(t)}{t-r} p_{\ell}(t) d \mu(t)=p_{\ell}(r) \int \frac{p_{n}(t)+\alpha p_{n-1}(t)}{t-r} d \mu(t) .
$$

In terms of the coefficients $c_{0}, \ldots, c_{n-1}$,

$$
c_{\ell} \int p_{\ell}(t)^{2} d \mu(t)=c_{0} p_{\ell}(r) \int d \mu(t) .
$$

Because the roots of $p_{n}+\alpha p_{n-1}$ and $p_{n-1}$ are interlaced, $r$ is greater than the largest root of $p_{n-1}$ and hence greater than the largest root of $p_{\ell}$. It follows that $p_{\ell}(r)>0$, and thus $c_{0}, \ldots, c_{n-1}$ all have the same sign. Comparing leading coefficients shows that $c_{n-1}=1$, so every coefficient must be positive.

There is a natural generalization of this proof to the case of arbitrary $k$ in Theorem 3.1. The key idea is to use orthogonality with respect to a signed measure, because for $k<n$,

$$
\prod_{i=1}^{k}\left(t-r_{i}\right)
$$

is the monic orthogonal polynomial of degree $k$ for the signed measure

$$
\left(t-r_{k+1}\right) \ldots\left(t-r_{n}\right) d \mu(t),
$$

assuming there is a unique such polynomial, as we prove below in Lemma 3.5 and Lemma 3.7. To verify that, we simply need to show that it is orthogonal to all polynomials of degree less than $k$, which is equivalent with the orthogonality of $p_{n}(t)+\alpha p_{n-1}(t)$ to all such polynomials with respect to $d \mu(t)$. (For $k=n$ the conclusion is false unless $\alpha=0$.) More generally, we have the following lemma:

Lemma 3.3. Suppose $\nu$ is a signed measure that has monic orthogonal polynomials $q_{0}, q_{1}, \ldots, q_{N+1}$, where $\operatorname{deg}\left(q_{i}\right)=i$. For $i \leq N$, if $q_{i}(r) \neq 0$ and $(t-r) d \nu(t)$ has a unique degree $i$ monic orthogonal polynomial, then it equals

$$
\frac{q_{i+1}(t)+\alpha_{i} q_{i}(t)}{t-r}
$$

where $\alpha_{i}$ is chosen so that $q_{i+1}(r)+\alpha_{i} q_{i}(r)=0$. 
Proof. The orthogonality of this polynomial with respect to $(t-r) d \nu(t)$ amounts to the orthogonality with respect to $d \nu(t)$ of $q_{i+1}(t)+\alpha_{i} q_{i}(t)$ to all polynomials of degree at most $i-1$.

One might hope to imitate the proof of Proposition 3.2 as one removes successive roots of $p_{n}+\alpha p_{n-1}$. In fact, this approach works, but it is not obvious that it works, because of a fundamental technical difficulty. As soon as one introduces linear factors such as $t-r$ into the measure, it is no longer a positive measure but rather a signed measure. Many of the basic properties of orthogonal polynomials fail in the case of a signed measure. Monic orthogonal polynomials may not exist or be unique, and the roots of $p_{i}$ and $p_{i+1}$ may not be interlaced. In addition, there is an even more dramatic problem: the proof of Proposition 3.2 depends on the positivity of

$$
\int p_{\ell}(t)^{2} d \mu(t)
$$

and

$$
\int d \mu(t)
$$

neither of which is generally positive for a signed measure.

Fortunately, the measures that actually arise in the proof will be considerably better behaved than a typical signed measure. In particular, they will all have the following property for some $N$ :

Definition 3.4. Let $\nu$ be a signed Borel measure on $\mathbb{R}$ with respect to which all polynomials are integrable. We say $\nu$ is positive definite up to degree $N$ if for all polynomials $f$ such that $\operatorname{deg}(f) \leq N$,

$$
\int f(t)^{2} d \nu(t) \geq 0
$$

with equality only if $f$ is identically zero.

If $\nu$ is positive definite up to degree $N$, then many of the usual properties hold for orthogonal polynomials of degrees up to $N+1$. Lemma 3.5 covers the properties we will require.

Lemma 3.5. Let $\nu$ be positive definite up to degree $N$. Then there are unique monic polynomials $q_{0}, q_{1}, \ldots, q_{N+1}$ such that $\operatorname{deg}\left(q_{i}\right)=i$ for each $i$ and

$$
\int q_{i}(t) q_{j}(t) d \nu(t)=0
$$

for $i \neq j$. For each $i, q_{i}$ has $i$ distinct real roots, and the roots of $q_{i}$ and $q_{i-1}$ are interlaced.

The standard proofs for orthogonal polynomials from Chapter 5 of AAR can be adapted, if one uses the trick of writing a nonnegative polynomial as a sum of two squares. Here we use instead a particularly elegant proof from [Si, p. 14]:

Proof. The orthogonal polynomials for $\nu$ are obtained by applying Gram-Schmidt orthogonalization to $1, t, \ldots, t^{N+1}$ :

$$
q_{i}(t)=t^{i}-\sum_{j=0}^{i-1} q_{j}(t) \frac{\int s^{i} q_{j}(s) d \nu(s)}{\int q_{j}(s)^{2} d \nu(s)},
$$


which makes sense for $i \leq N+1$ because the norm of $q_{j}$ in the denominator is always nonzero. They are unique because the quotients of integrals are the only coefficients in such an expansion that yield orthogonality.

The next step is to prove the three-term recurrence relation. The polynomial $q_{i}(t)-t q_{i-1}(t)$ is orthogonal to all polynomials of degree less than $i-2$, and hence there exist constants $a_{i}$ and $b_{i}$ such that

$$
q_{i}(t)=\left(t+a_{i}\right) q_{i-1}(t)+b_{i} q_{i-2}(t) .
$$

Multiplying by $q_{i-2}(t)$ and integrating yields

$$
b_{i} \int q_{i-2}(t)^{2} d \nu(t)=-\int q_{i-1}(t) t q_{i-2}(t) d \nu(t),
$$

by orthogonality. The polynomials $t q_{i-2}(t)$ and $q_{i-1}(t)$ differ by a polynomial of degree at most $i-2$, and hence

$$
\int q_{i-1}(t) t q_{i-2}(t) d \nu(t)=\int q_{i-1}(t)^{2} d \nu(t)
$$

Combining the last two equations shows that $b_{i}<0$.

We now prove by induction that the roots of $q_{i}$ and $q_{i-1}$ are real and interlaced. The base case of $i=1$ is trivial. For the induction step, suppose the roots of $q_{i-1}$ and $q_{i-2}$ are real and interlaced. For each root $r$ of $q_{i-1}$,

$$
q_{i}(r)=b_{i} q_{i-2}(r),
$$

by the three-term recurrence relation. By hypothesis, $q_{i-2}$ alternates in sign at the roots of $q_{i-1}$. Because $b_{i}$ is negative, $q_{i}$ and $q_{i-2}$ have opposite signs at the roots of $q_{i-1}$. That implies that $q_{i}$ must have a root between each pair of consecutive roots of $q_{i-1}$. Because $q_{i}(t)$ and $q_{i-2}(t)$ have the same sign when $|t|$ is sufficiently large, $q_{i}$ must also have a root greater than every root of $q_{i-1}$ and a root less than every root of $q_{i-1}$. All $i$ roots of $q_{i}$ are now accounted for, so all the roots of $q_{i}$ are real and interlaced with those of $q_{i-1}$. That completes the proof.

The following minor variant of Gauss-Jacobi quadrature (see Theorem 5.3.2 in AAR ) will prove crucial for understanding the signed measures that arise in our proof.

Lemma 3.6. Let $\alpha$ be any real number, and let

$$
r_{1}<r_{2}<\cdots<r_{n}
$$

be the roots of $p_{n}+\alpha p_{n-1}$. Then there are positive numbers $\lambda_{1}, \ldots, \lambda_{n}$ such that for every polynomial $f$ of degree at most $2 n-2$,

$$
\int f(t) d \mu(t)=\sum_{i=1}^{n} \lambda_{i} f\left(r_{i}\right)
$$

Proof. There exist coefficients $\lambda_{1}, \ldots, \lambda_{n}$ such that

$$
\int f(t) d \mu(t)=\sum_{i=1}^{n} \lambda_{i} f\left(r_{i}\right)
$$

whenever $\operatorname{deg}(f)<n$, because a polynomial of degree less than $n$ is completely determined by its values at $r_{1}, \ldots, r_{n}$ and the map taking these values to the integral is linear. 
For $\operatorname{deg}(f) \leq 2 n-2$, there exist polynomials $g$ and $h$ satisfying

$$
f=\left(p_{n}+\alpha p_{n-1}\right) g+h
$$

with $\operatorname{deg}(g) \leq n-2$ and $\operatorname{deg}(h)<n$. By orthogonality,

$$
\int f(t) d \mu(t)=\int h(t) d \mu(t)
$$

and $f\left(r_{i}\right)=h\left(r_{i}\right)$ for each $i$ because $p_{n}\left(r_{i}\right)+\alpha p_{n-1}\left(r_{i}\right)=0$ by definition. It follows that

$$
\int f(t) d \mu(t)=\sum_{i=1}^{n} \lambda_{i} f\left(r_{i}\right)
$$

holds whenever $\operatorname{deg}(f) \leq 2 n-2$.

All that remains is to prove positivity for the coefficients. For fixed $i$, let

$$
f(t)=\prod_{j \neq i}\left(t-r_{j}\right)^{2} .
$$

Then

$$
\int f(t) d \mu(t)=\lambda_{i} f\left(r_{i}\right)^{2}
$$

from which it follows that $\lambda_{i}>0$.

Let $r_{1}<\cdots<r_{n}$ be the roots of $p_{n}+\alpha p_{n-1}$, and for $0 \leq j \leq n$ define the measure $\mu_{j}$ by

$$
d \mu_{j}(t)=\prod_{i=0}^{j-1}\left(r_{n-i}-t\right) d \mu(t)
$$

(of course $\mu_{0}=\mu$ ).

Lemma 3.7. For $0 \leq j \leq n-1$, the measure $\mu_{j}$ is positive definite up to degree $n-j-1$.

Proof. If $\operatorname{deg}(f) \leq n-1-j / 2$, then by Lemma 3.6.

$$
\int f(t)^{2} \prod_{i=0}^{j-1}\left(r_{n-i}-t\right) d \mu(t)=\sum_{i=1}^{n} \lambda_{i} f\left(r_{i}\right)^{2} \prod_{\ell=0}^{j-1}\left(r_{n-\ell}-r_{i}\right) \geq 0 ;
$$

equality holds if and only if $f$ vanishes at $r_{1}, \ldots, r_{n-j}$, which is impossible for a polynomial of degree at most $n-j-1$ unless it vanishes identically.

Let $q_{j, i}$ be the monic orthogonal polynomial of degree $i$ for $\mu_{j}$. (Note that $q_{0, i}=p_{i}$.) The polynomial $q_{j, i}$ is simplest when $i=n-j$, in which case we have

$$
q_{j, n-j}(t)=\left(t-r_{1}\right) \ldots\left(t-r_{n-j}\right),
$$

as pointed out earlier. That implies that for $i<n-j$, the largest root of $q_{j, i}$ is less than $r_{n-j}$, because the roots are interlaced.

Equivalently, for $i \leq n-j$, the largest root of $q_{j-1, i}$ is less than $r_{n-j+1}$, so $q_{j-1, i}\left(r_{n-j+1}\right) \neq 0$. Hence Lemma 3.3 implies that for $i \leq n-j$, there are constants $\alpha_{j, i}$ such that

$$
q_{j, i}(t)=\frac{q_{j-1, i+1}(t)+\alpha_{j, i} q_{j-1, i}(t)}{t-r_{n-j+1}} .
$$

Lemma 3.8. For $1 \leq j \leq n$ and $i \leq n-j$, the polynomial $q_{j, i}$ is a positive linear combination of $q_{j-1,0}, \ldots, q_{j-1, i}$. 
Proof. Define $c_{0}, \ldots, c_{i}$ so that

$$
q_{j, i}(t)=\sum_{\ell=0}^{i} c_{\ell} q_{j-1, \ell}(t) .
$$

We argue as in the proof of Proposition 3.2, For $\ell \leq i$,

$$
\int\left(q_{j-1, i+1}(t)+\alpha_{j, i} q_{j-1, i}(t)\right) \frac{q_{j-1, \ell}(t)-q_{j-1, \ell}\left(r_{n-j+1}\right)}{t-r_{n-j+1}} d \mu_{j-1}(t)=0
$$

by orthogonality, because

$$
\frac{q_{j-1, \ell}(t)-q_{j-1, \ell}\left(r_{n-j+1}\right)}{t-r_{n-j+1}}
$$

is a polynomial of degree $\ell-1$, which is less than $i$. It now follows from

$$
q_{j, i}(t)=\frac{q_{j-1, i+1}(t)+\alpha_{j, i} q_{j-1, i}(t)}{t-r_{n-j+1}}
$$

and (3.1) that

$$
\int q_{j, i}(t) q_{j-1, \ell}(t) d \mu_{j-1}(t)=q_{j-1, \ell}\left(r_{n-j+1}\right) \int q_{j, i}(t) d \mu_{j-1}(t) .
$$

Thus,

$$
c_{\ell} \int q_{j-1, \ell}(t)^{2} d \mu_{j-1}(t)=c_{0} q_{j-1, \ell}\left(r_{n-j+1}\right) \int d \mu_{j-1}(t) .
$$

Because $\ell \leq i \leq n-j$, both integrals are positive by Lemma 3.7. The largest root of $q_{j-1, \ell}$ is less than $r_{n-j+1}$, so

$$
q_{j-1, \ell}\left(r_{n-j+1}\right)>0 .
$$

Thus, $c_{0}, \ldots, c_{i}$ all have the same sign, which is positive because $c_{i}=1$.

Theorem 3.1 follows from applying Lemma 3.8 repeatedly, starting with $q_{k, n-k}$.

\section{LiNEAR PROGRAMMING BOUNDS}

Theorem 1.2 is deduced from the following proposition, which originates in $\mathrm{Y}$ ] and is the key technique in $\mathrm{Y}$, KY1, KY2, An1, An2, (here we give a somewhat different proof from the one in $[\mathrm{Y}$, along the lines of [An2]):

Proposition 4.1. Let $f:(0,4] \rightarrow \mathbb{R}$ be any function. Suppose $h:[-1,1] \rightarrow \mathbb{R}$ is a polynomial such that

$$
h(t) \leq f(2-2 t)
$$

for all $t \in[-1,1)$, and suppose there are nonnegative coefficients $\alpha_{0}, \ldots, \alpha_{d}$ such that $h$ has the expansion

$$
h(t)=\sum_{i=0}^{d} \alpha_{i} C_{i}^{n / 2-1}(t)
$$

in terms of ultraspherical polynomials. Then every set of $N$ points on $S^{n-1}$ has potential energy at least

$$
N^{2} \alpha_{0}-N h(1)
$$

with respect to the potential function $f$. 
Fundamentally, this proposition is a generalization of the linear programming bounds for spherical codes due independently to Kabatiansky and Levenshtein in KL and Delsarte, Goethals, and Seidel in [DGS] (see also Chapter 9 in [CS]). As is pointed out in $[\mathrm{Y}$, one can derive those bounds for spherical codes with minimal angle $\theta$ from Proposition 4.1 by setting

$$
f(2-2 t)= \begin{cases}\infty & \text { if } t>\cos \theta, \text { and } \\ 0 & \text { otherwise }\end{cases}
$$

(Of course, $f$ takes values in $\mathbb{R} \cup\{\infty\}$, but that is not a problem.) The potential energy is then 0 for a spherical code with minimal angle at least $\theta$ and $\infty$ otherwise; Proposition 4.1 implies that no such code can exist if $N>h(1) / \alpha_{0}$.

Choosing the optimal function $h$ amounts to solving an infinite-dimensional linear programming problem: the potential energy bound is a linear functional of $h$, and the only restrictions on $h$ are the linear constraints on its values and ultraspherical coefficients. As in the case of the traditional linear programming bounds, it is not hard to approximate the optimum numerically (by solving a finite-dimensional linear programming problem in which one imposes the constraint $h(t) \leq f(2-2 t)$ for only finitely many values of $t)$. Even though the bound is usually not sharp, it is often quite close. For example, for twenty points on $S^{2}$ and the Coulomb potential function $f(r)=1 / r^{1 / 2}$, the minimal-energy configuration, which incidentally is not the vertices of a regular dodecahedron, appears to have potential energy $301.763 \ldots$ The polynomial

$$
\begin{aligned}
h(t)= & 0.8729 C_{0}^{1 / 2}(t)+0.634 C_{1}^{1 / 2}(t)+0.425 C_{2}^{1 / 2}(t)+0.258 C_{3}^{1 / 2}(t) \\
& +0.135 C_{4}^{1 / 2}(t)+0.0569 C_{5}^{1 / 2}(t)+0.016 C_{6}^{1 / 2}(t)
\end{aligned}
$$

proves a lower bound of 301.204. It is not the best possible choice of $h$, but we have found no polynomial that beats 301.5.

What is remarkable is that in the case of the sharp arrangements, for every completely monotonic potential function there is an auxiliary function that proves a sharp bound, as we show in the proof of Theorem 1.2 .

Proof of Proposition 4.1. Suppose $\mathcal{C} \subset S^{n-1}$ has cardinality $N$. By assumption,

$$
\sum_{x, y \in \mathcal{C}, x \neq y} f\left(|x-y|^{2}\right) \geq \sum_{x, y \in \mathcal{C}, x \neq y} h(\langle x, y\rangle),
$$

because $|x-y|^{2}=2-2\langle x, y\rangle$. On the other hand,

$$
\begin{aligned}
\sum_{x, y \in \mathcal{C}, x \neq y} h(\langle x, y\rangle) & =-N h(1)+\sum_{x, y \in \mathcal{C}} h(\langle x, y\rangle) \\
& =-N h(1)+\sum_{i=0}^{d} \alpha_{i} \sum_{x, y \in \mathcal{C}} C_{i}^{n / 2-1}(\langle x, y\rangle)
\end{aligned}
$$

Because $C_{i}^{n / 2-1}$ is a positive-definite kernel (see Subsection 2.2),

$$
\sum_{x, y \in \mathcal{C}} C_{i}^{n / 2-1}(\langle x, y\rangle) \geq 0
$$


It follows that

$$
\sum_{x, y \in \mathcal{C}, x \neq y} h(\langle x, y\rangle) \geq-N h(1)+\alpha_{0} \sum_{x, y \in \mathcal{C}} C_{0}^{n / 2-1}(\langle x, y\rangle)=N^{2} \alpha_{0}-N h(1),
$$

as desired.

The bound proved by an auxiliary polynomial $h$ is sharp for a configuration $\mathcal{C}$ and potential function $f$ if and only if two conditions hold: $h(t)$ must equal $f(2-2 t)$ at every inner product $t$ that occurs between distinct points in $\mathcal{C}$, and whenever the ultraspherical coefficient $\alpha_{i}$ is positive with $i>0$, we must have

$$
\sum_{x, y \in \mathcal{C}} C_{i}^{n / 2-1}(\langle x, y\rangle)=0
$$

In particular, if $h$ is strictly positive definite, then $\mathcal{C}$ must be a spherical $\operatorname{deg}(h)$ design, by Theorem 5.5 in DGS.

One can easily generalize Proposition 4.1 to deal with the case of unequally charged particles (this was pointed out in Theorem 1 of $[\mathrm{Y}]$ ). Suppose we have $N$ real numbers $c_{1}, \ldots, c_{N}$, which we refer to as charges; they must all have the same sign. Given a potential function $f$ and an ordered $N$-tuple $\left(x_{1}, \ldots, x_{N}\right) \in\left(S^{n-1}\right)^{N}$, define the potential energy to be

$$
\sum_{1 \leq i, j \leq N, i \neq j} c_{i} c_{j} f\left(\left|x_{i}-x_{j}\right|^{2}\right) .
$$

Under the conditions in Proposition 4.1, no such configuration can have potential energy less than

$$
\left(\sum_{i=1}^{N} c_{i}\right)^{2} \alpha_{0}-\left(\sum_{i=1}^{N} c_{i}^{2}\right) h(1) .
$$

We know of few beautiful configurations with unequal charges and none for which linear programming bounds are sharp, but they may well exist.

\section{Choosing auxiliary functions}

In this section, we describe an explicit choice for the auxiliary function $h$ in Proposition 4.1] it is analogous to the constructions using the Christoffel-Darboux formula in $\mathrm{KL}$, MRRW]. We will not require this function later in the paper, but it provides a simple way to apply Proposition 4.1, and the techniques used in analyzing it will be important in the proof of Theorem 1.2 .

Let $f:(0,4] \rightarrow \mathbb{R}$ be completely monotonic. From the proof of Proposition 4.1 we know that it is more natural to look at $a(t)=f(2-2 t)$, because $|x-y|^{2}=2-2\langle x, y\rangle$. The function $a$ is absolutely monotonic on $[-1,1)$ because $f$ is completely monotonic on $(0,4]$, and $a$ is strictly absolutely monotonic if and only if $f$ is strictly completely monotonic.

Our construction of $h$ requires three inputs: the dimension $n-1$ of the sphere $S^{n-1}$, a natural number $m \geq 1$, and a real number $\alpha \geq 0$. Let

$$
t_{1}<\cdots<t_{m}
$$

be the roots of the polynomial $C_{m}^{n / 2-1}+\alpha C_{m-1}^{n / 2-1}$. We require that $t_{1} \geq-1$ and thus $\left\{t_{1}, \ldots, t_{m}\right\} \subset[-1,1)$; by Theorem 3.3.4 in $\mathrm{Sz}$, that amounts to $\alpha \leq$ $-C_{m}^{n / 2-1}(-1) / C_{m-1}^{n / 2-1}(-1)$ (which is positive because $C_{i}^{n / 2-1}(-1)$ has sign $\left.(-1)^{i}\right)$. 
Let $h(t)$ be the Hermite interpolating polynomial that agrees with $a(t)$ to order 2 at each $t_{i}$ (i.e., $h\left(t_{i}\right)=a\left(t_{i}\right)$ and $h^{\prime}\left(t_{i}\right)=a^{\prime}\left(t_{i}\right)$ ). The idea of constructing auxiliary functions via Hermite interpolation originates in $[\mathrm{Y}]$.

Lemma 5.1. For all $t \in[-1,1)$,

$$
h(t) \leq a(t) .
$$

Proof. By Lemma 2.1, there exists a point $\xi$ such that

$$
\min \left(t, t_{1}, \ldots, t_{m}\right)<\xi<\max \left(t, t_{1}, \ldots, t_{m}\right)
$$

and

$$
a(t)-h(t)=\frac{a^{(2 m)}(\xi)}{(2 m) !}\left(t-t_{1}\right)^{2} \ldots\left(t-t_{m}\right)^{2} .
$$

The right side is nonnegative, and hence $a(t) \geq h(t)$.

The last requirement before we can apply Proposition 4.1, namely that $h$ must be a positive-definite function, is more subtle. Let

$$
F(t)=C_{m}^{n / 2-1}(t)+\alpha C_{m-1}^{n / 2-1}(t)=\prod_{i=1}^{m}\left(t-t_{i}\right) .
$$

In the notation introduced before Proposition [2.2, $h=H\left(a, F^{2}\right)$. We will show that $F^{2}$ has a strong property that we refer to as conductivity:

Definition 5.2. A nonconstant polynomial $g$ with all its roots in $[-1,1)$ is conductive if for all absolutely monotonic functions $a$ on $[-1,1), H(a, g)$ is positive definite. It is strictly conductive if it is conductive and for all strictly absolutely monotonic $a, H(a, g)$ is strictly positive definite of degree $\operatorname{deg}(g)-1$.

Lemma 5.3. If $g_{1}$ and $g_{2}$ are conductive and $g_{1}$ is positive definite, then $g_{1} g_{2}$ is conductive. If $g_{1}$ is conductive and strictly positive definite and $g_{2}$ is strictly conductive, then $g_{1} g_{2}$ is strictly conductive.

Proof. As noted in the proof of Proposition 2.2, for every absolutely monotonic function $a$ on $[-1,1)$,

$$
H\left(a, g_{1} g_{2}\right)=H\left(a, g_{1}\right)+g_{1} H\left(Q\left(a, g_{1}\right), g_{2}\right) .
$$

The lemma now follows from Proposition 2.2 and the fact that the set of positivedefinite (or strictly positive-definite) functions is closed under taking products.

For $r \in[-1,1)$, let $\ell_{r}$ denote the linear polynomial $\ell_{r}(t)=t-r$. Clearly $\ell_{r}$ is conductive, and even strictly conductive if $r \neq-1$, because $H\left(a, \ell_{r}\right)$ is the constant polynomial $a(r)$, which is nonnegative. Lemma 5.3 implies that if $g$ is conductive and positive definite, then $g \ell_{r}$ is conductive.

Consider the partial products

$$
\prod_{i=1}^{j}\left(t-t_{i}\right)
$$

for $j \leq m$. By Theorem 3.1, this product is positive definite for $j<m$ (even strictly positive definite), and for $j=m$ it is positive definite because $\alpha \geq 0$. It follows 
from Lemma 5.3 and the remarks in the previous paragraph that each product is conductive. Thus,

$$
\prod_{i=1}^{m}\left(t-t_{i}\right)
$$

is conductive, so Lemma 5.3 implies that its square is as well. Because $h=$ $H\left(a, F^{2}\right)$, we conclude that $h$ is positive definite, as desired.

These auxiliary polynomials are rarely optimal. For example, consider the case $n=3, N=20$, and $f(r)=1 / r^{1 / 2}$ that was discussed in Section 4 Numerical calculations suggest that the best bound is obtained by taking $m=3$ and $\alpha=$ $0.752718117 \ldots$ (an algebraic number of degree 22). That yields a lower bound of 299.708 . . , which is worse than the bound of 301.204 derived in Section 4 . However, it is reasonably close, especially given how simple and systematic the construction of $h$ was.

The connection between our construction and those used in [MRRW] and [KL] may not be immediately apparent. Those constructions are equivalent to using the polynomial

$$
\left(t-t_{m}\right) \prod_{i=1}^{m-1}\left(t-t_{i}\right)^{2}
$$

in the linear programming bounds for spherical codes with minimal angle $\theta$ satisfying $\cos \theta=t_{m}$. The fact that

$$
\prod_{i=1}^{m-1}\left(t-t_{i}\right)
$$

is positive definite is proved in those papers using the Christoffel-Darboux formula. Unlike in our construction, $\alpha$ can be arbitrarily large.

One can generalize the construction from this section as follows. Let $p_{0}, p_{1}, \ldots$ be any family of orthogonal polynomials such that each is positive definite. Given $m$ and $\alpha$, choose $t_{1}, \ldots, t_{m}$ to be the roots of $p_{m}+\alpha p_{m-1}$. The construction and proof are essentially the same as before. The proof of Theorem 1.2 will be based on this approach.

\section{Proof of the Main theorem}

Let $f:(0,4] \rightarrow \mathbb{R}$ be completely monotonic, define $a(t)=f(2-2 t)$ as in Section 5 , and let $\mathcal{C} \subset S^{n-1}$ be a sharp arrangement with $|\mathcal{C}|=N$. To prove Theorem 1.2. we will construct an auxiliary polynomial $h$ that satisfies the hypotheses of Proposition 4.1 and proves a sharp bound. The case when $\mathcal{C}$ is the set of vertices of the 600 -cell is qualitatively more difficult than the other cases, so for the time being we assume that we are in the sharp case. (We deal with the 600-cell in Section 7.)

Suppose that the possible inner products that occur between distinct points in $\mathcal{C}$ are $t_{1}, \ldots, t_{m}$, ordered so that

$$
-1 \leq t_{1}<\cdots<t_{m}<1 .
$$

Let $h(t)$ be the Hermite interpolating polynomial that agrees with $a(t)$ to order 2 at each $t_{i}$.

When $t_{1}=-1$, it might seem more natural to interpolate only to first order at that point. The purpose of second-order interpolation is to avoid sign changes in $h(t)-a(t)$ when $t=t_{i}$, and that is not a concern when $t=-1$. In fact, firstorder interpolation works there, but we will omit the details here, because the only 
advantage we see in it is that it leads to a slightly improved bound on the number of derivative sign conditions one must impose on the potential function (it decreases by 1 the bound $K$ mentioned in Section 11). See Section 8 for the details of that approach in a context in which it is more useful.

Lemma 6.1. For all $t \in[-1,1)$,

$$
h(t) \leq a(t) .
$$

The proof is identical to that of Lemma 5.1 Thus, the first hypothesis of Proposition 4.1 is satisfied. What remains to be shown is that $h$ is a positive-definite function and that the bound is sharp. (The bound derived from this function $h$ is not sharp for the 600-cell, which is why that case is more subtle and we have postponed it until later.)

The sharpness of the lower bound is simple, because it follows from the definition of a sharp arrangement that $\mathcal{C}$ is a spherical $\operatorname{deg}(h)$-design. Thus,

$$
\sum_{x, y \in \mathcal{C}} C_{i}^{n / 2-1}(\langle x, y\rangle)=0
$$

whenever $0<i \leq \operatorname{deg}(h)$; in fact, for each fixed $y$, the polynomial $C_{i}^{n / 2-1}(\langle x, y\rangle)$ has the same average over $\mathcal{C}$ as over the entire sphere $S^{n-1}$, and it integrates to 0 over the sphere because it is a positive-degree spherical harmonic and thus orthogonal to the constant functions. Because $h$ agrees with $a$ at the inner products $t_{1}, \ldots, t_{m}$ from $\mathcal{C}$, the conditions for a sharp bound that were listed after the proof of Proposition 4.1 are satisfied.

The proof that $h$ is positive definite is analogous to the proof in Section 5, but it will involve more elaborate lemmas. Define

$$
F(t)=\prod_{i=1}^{m}\left(t-t_{i}\right)
$$

Then $h=H\left(a, F^{2}\right)$, and we will prove that $F^{2}$ is conductive. In fact, we will show that $F^{2}$ is strictly conductive, which will be important in the proof of uniqueness.

Lemma 6.2. The function $F$ is strictly positive definite.

This lemma is a standard fact in the theory of linear programming bounds. We prove it as in [El].

Proof. The leading coefficient of $F$ in terms of powers of $t$ is positive, which implies that its leading ultraspherical coefficient is also positive. For the others, we apply orthogonality to see that the $i$-th ultraspherical coefficient of $F$ equals a positive constant (depending on $i$ ) times

$$
\int_{S^{n-1}} F(\langle x, y\rangle) C_{i}^{n / 2-1}(\langle x, y\rangle) d x
$$

where $y$ is an arbitrary point on $S^{n-1}$ and of course $d x$ denotes surface measure on $S^{n-1}$. Choose $y \in \mathcal{C}$.

Because we know that the leading ultraspherical coefficient in $F$ is positive, we can take $i<\operatorname{deg}(F)$. Thus, $\operatorname{deg}(F)+i \leq 2 \operatorname{deg}(F)-1$. Because $\mathcal{C}$ is a sharp 
configuration, it is a spherical $(2 \operatorname{deg}(F)-1)$-design. It follows that the integral above equals a positive constant times

$$
\sum_{x \in \mathcal{C}} F(\langle x, y\rangle) C_{i}^{n / 2-1}(\langle x, y\rangle) .
$$

By the construction of $F$, each term in the sum vanishes except for $x=y$, so the sum equals $F(1) C_{i}^{n / 2-1}(1)$. Both factors are positive, as desired.

In order to deal with the partial products

$$
\prod_{i=1}^{j}\left(t-t_{i}\right)
$$

we must express $F$ in terms of orthogonal polynomials. Recall that the ultraspherical polynomials $C_{i}^{n / 2-1}$ are orthogonal with respect to the measure

$$
\left(1-t^{2}\right)^{(n-3) / 2} d t
$$

on $[-1,1]$. Call that measure $d \mu(t)$. Let $p_{0}, p_{1}, \ldots$ denote the monic orthogonal polynomials with respect to $(1-t) d \mu(t)$. These polynomials are a special case of Jacobi polynomials, but we will require only one fact about them: they are nonnegative linear combinations of the ultraspherical polynomials $C_{i}^{n / 2-1}$. That follows from Lemma 3.3 and Proposition 3.2 .

Lemma 6.3. There exists a constant $\alpha$ such that $F=p_{m}+\alpha p_{m-1}$.

Proof. We simply need to show that $F$ is orthogonal to all polynomials of degree at most $m-2$ with respect to the measure $(1-t) d \mu(t)$. That is equivalent to showing that $(1-t) F(t)$ is orthogonal to all such polynomials with respect to $d \mu(t)$.

Let $p$ be any polynomial of degree at most $m-2$. Because $\mathcal{C}$ is a sharp configuration, it must be a spherical $(2 m-1)$-design. It follows as in the previous proof that

equals a positive linear combination of

$$
\int(1-t) F(t) p(t) d \mu(t)
$$

$$
\left(1-t_{1}\right) F\left(t_{1}\right) p\left(t_{1}\right), \ldots,\left(1-t_{m}\right) F\left(t_{m}\right) p\left(t_{m}\right),(1-1) F(1) p(1) .
$$

Each of these vanishes because $F$ vanishes at $t_{1}, \ldots, t_{m}$. Thus,

$$
\int F(t) p(t)(1-t) d \mu(t)=0
$$

as desired.

Combining Lemma 6.3 with Theorem 3.1 shows that for $j<m$, the polynomial

$$
\prod_{i=1}^{j}\left(t-t_{i}\right)
$$

is strictly positive definite, and the case $j=m$ is Lemma 6.2. Now the proof that $F$ and $F^{2}$ are conductive concludes as in Section 5 , by using Lemma 5.3 repeatedly.

We must still prove the additional uniqueness results when $f$ is strictly completely monotonic. For that, we use the following lemma:

Lemma 6.4. If a satisfies $a^{(k)}(t)>0$ for all $k \geq 0$ and $t \in(-1,1)$, then $a(t)-h(t)$ has at most $\operatorname{deg}(h)+1$ roots in $[-1,1)$, counted with multiplicity. 
Proof. By Rolle's theorem, $a(t)-h(t)$ has at most one more root than $a^{\prime}(t)-$ $h^{\prime}(t)$. Repeated differentiation reduces to the case in which $\operatorname{deg}(h)=0$, which is trivial.

Thus, the only roots of $a(t)-h(t)$ in $[-1,1)$ are at $t=t_{i}$ for some $i$. It follows from the proof of Proposition 4.1 that if $\mathcal{C}^{\prime}$ is any other potential energy minimum with $\left|\mathcal{C}^{\prime}\right|=|\mathcal{C}|$, then all inner products between distinct points of $\mathcal{C}^{\prime}$ occur among $t_{1}, \ldots, t_{m}$. Thus $\mathcal{C}^{\prime}$ is a spherical code with the same parameters as $\mathcal{C}$, and each sharp configuration listed in Table 1 except on the last line is known to be the unique spherical code with its parameters, up to orthogonal transformations, of course. See Appendix $\mathrm{A}$ for details and references.

Even when the spherical code is not unique, one can still conclude from the strict absolute monotonicity of $a$ that $\mathcal{C}^{\prime}$ must be sharp. The key observation is that $F^{2}$ is strictly conductive (except in the trivial case when $\mathcal{C}$ consists of two antipodal points), which follows easily from Lemma 5.3. It implies that $h$ is a strictly positivedefinite polynomial of degree $2 m-1$. Then the sharpness in Proposition 4.1 implies that $\mathcal{C}^{\prime}$ must be a spherical $\operatorname{deg}(h)$-design (by Theorem 5.5 in [DGS) and hence a sharp configuration. Furthermore, each of $t_{1}, \ldots, t_{m}$ must occur as an inner product in $\mathcal{C}^{\prime}$, from switching the roles of $\mathcal{C}$ and $\mathcal{C}^{\prime}$.

\section{THE 600-CELL}

The final configuration is the vertices of the regular 600-cell. The construction in Section 6 does not work in this case, and overall the 600-cell appears to be intrinsically more complicated than the sharp configurations. The fundamental problem is that it is only a spherical 11-design, but the polynomial $h$ constructed as in the previous section would have degree 15 (or 14 if one uses the alternate construction for antipodal configurations). Recall that being a spherical $\operatorname{deg}(h)$ design was crucial to having a sharp bound. In fact, $h$ does turn out to be a positive-definite function in this case, but it proves a suboptimal bound.

Andreev and Elkies have shown in [An3, El] that a degree 17 polynomial with coefficients in $\mathbb{Q}(\sqrt{5})$ proves a sharp bound for the number of points in a spherical code with minimal angle $\pi / 5=\cos ^{-1}(1+\sqrt{5}) / 4$ on $S^{3}$, although polynomials of lower degree do not work. A similar phenomenon occurs in our problem. What saves the proof is that although the 600 -cell is not a spherical 12-design, all spherical harmonics of degrees from 13 to 19 do indeed sum to 0 over the 600-cell. Degree 12 is the only problem, and that can be addressed by ensuring that the 12-th ultraspherical coefficient of $h$ vanishes. The fact that spherical harmonics of degrees 13 through 19 sum to 0 over the 600-cell is easily checked using the distance distribution from Table 2, which tells for each vertex how many others have a given inner product with it. One can also prove it using invariant theory as follows. The symmetry group of the 600-cell is the $H_{4}$ reflection group, for which the ring of invariant polynomials has generators of degrees 2 (the trivial invariant $x \mapsto|x|^{2}$ ), 12, 20, and 30. Summing a polynomial over the vertices of the 600-cell amounts to averaging it with respect to $H_{4}$. For any degree that is not a nonnegative integer combination of 12,20 , and 30 , averaging must yield a polynomial that is constant on $S^{3}$, and that constant vanishes for spherical harmonics because they are orthogonal to the constant polynomials.

For the 600-cell, we have $m=8$ and $\left\{t_{1}, \ldots, t_{m}\right\}=\{-1,0, \pm 1 / 2,( \pm 1 \pm \sqrt{5}) / 4\}$; we order the inner products so that $t_{1}<\cdots<t_{8}$. Let $h(t)$ be the unique polynomial 
TABLE 2. The distance distribution of the 600-cell.

\begin{tabular}{cc} 
Inner Product & Count \\
\hline \pm 1 & 1 \\
$( \pm 1 \pm \sqrt{5}) / 4$ & 12 \\
$\pm 1 / 2$ & 20 \\
0 & 30
\end{tabular}

of degree at most 17 such that $h\left(t_{i}\right)=a\left(t_{i}\right)$ for $1 \leq i \leq 8, h^{\prime}\left(t_{i}\right)=a^{\prime}\left(t_{i}\right)$ for $2 \leq i \leq 8$, and $\alpha_{11}=\alpha_{12}=\alpha_{13}=0$, where $\alpha_{i}$ denotes the $i$-th ultraspherical coefficient of $h$. Note that we do not require that $h^{\prime}(-1)=a^{\prime}(-1)$.

It might seem more natural to use a polynomial of degree 15 and simply set $\alpha_{12}=0$, but that does not work. We have no conceptual explanation for why it fails or why our slightly more complicated approach succeeds.

If $h(t) \leq a(t)$ for all $t$ and $h$ is positive definite, then it proves a sharp bound (as pointed out above, because $\alpha_{12}=0$ ). Unfortunately, we know of no simple reason why these inequalities hold. We have proved them using a computer algebra system. The proof is completely rigorous, in the sense that it uses no floating point arithmetic or other unrigorous approximations; all computations are performed using exact arithmetic in $\mathbb{Q}(\sqrt{5})$.

7.1. Proof that $h$ is positive definite. We wish to prove that $h(t)$ is a nonnegative linear combination of the ultraspherical polynomials $C_{i}^{1}(t)$. Each ultraspherical coefficient of $h(t)$ is a linear function of $a\left(t_{i}\right)$ and $a^{\prime}\left(t_{i}\right)$ for $1 \leq i \leq m$. Let $u_{i}$ denote the coefficient of $a\left(t_{i}\right)$ in this linear function, and let $v_{i}$ denote the coefficient of $a^{\prime}\left(t_{i}\right)$.

As explained in Section 1, it suffices to consider the potential functions $f(r)=$ $(4-r)^{k}$ with $k \in\{0,1,2, \ldots\}$; up to scaling, that amounts to taking $a(t)=(1+t)^{k}$. The linear combination

$$
\sum_{i=1}^{m}\left(u_{i} a\left(t_{i}\right)+v_{i} a^{\prime}\left(t_{i}\right)\right)
$$

then becomes

$$
\sum_{i=1}^{m}\left(u_{i}\left(1+t_{i}\right)^{k}+v_{i} k\left(1+t_{i}\right)^{k-1}\right) .
$$

If $v_{8}>0$, then this expression is positive for all sufficiently large $k$. To prove that it is nonnegative for all $k$, we simply compute a bound $\ell$ such that it is guaranteed to be positive for all $k \geq \ell$ and then check nonnegativity for $0 \leq k<\ell$.

Let

$$
\chi(x)= \begin{cases}x & \text { if } x \leq 0, \text { and } \\ 0 & \text { if } x>0\end{cases}
$$

If $\ell$ is chosen so that

$$
\sum_{i=1}^{m}\left(\chi\left(u_{i}\right)\left(1+t_{i}\right)^{\ell}+\chi\left(v_{i}\right) \ell\left(1+t_{i}\right)^{\ell-1}\right)+v_{8} \ell\left(1+t_{8}\right)^{\ell-1} \geq 0
$$


then

$$
\sum_{i=1}^{m}\left(u_{i}\left(1+t_{i}\right)^{k}+v_{i} k\left(1+t_{i}\right)^{k-1}\right) \geq 0
$$

for all $k \geq \ell$. In other words, once the asymptotically dominant term outweighs all the negative terms, it continues to do so forever (because whenever $k$ increases, the dominant term increases by a larger factor than any other term does).

To prove that $h$ is positive definite, we deal with eighteen cases, one for each ultraspherical coefficient of $h$. In each case, we compute the coefficients

$$
u_{1}, \ldots, u_{8}, v_{1}, \ldots, v_{8}
$$

explicitly as elements of $\mathbb{Q}(\sqrt{5})$. We then check that $v_{8}>0$ and that (7.1) holds with $\ell=32$. Finally, we check that the ultraspherical coefficient is nonnegative for $k \in\{0,1, \ldots, 31\}$. These calculations are too cumbersome to carry out by hand, but they are easily done using a computer algebra system.

7.2. Proof that $h(t) \leq a(t)$. Unfortunately, the inequality $h(t) \leq a(t)$ no longer has as simple a proof as in the case of the sharp configurations. To prove it, we will use Proposition 2.2, Let

$$
F(t)=(t+1) \prod_{i=2}^{m}\left(t+t_{i}\right)^{2},
$$

and let $\tilde{h}$ be the usual Hermite interpolation $H(a, F)$ of $a$ (without requiring any ultraspherical coefficients to vanish). By Proposition 2.2,

$$
\frac{a(t)-\tilde{h}(t)}{F(t)}
$$

is absolutely monotonic on $[-1,1)$. Let $q(t)$ be the quadratic Taylor polynomial for this quotient about $t=-1$. Then it follows from absolute monotonicity that

$$
\frac{a(t)-\tilde{h}(t)}{F(t)} \geq q(t)
$$

for all $t \in[-1,1)$. Thus,

$$
\frac{a(t)-h(t)}{F(t)} \geq q(t)+\frac{\tilde{h}(t)-h(t)}{F(t)} .
$$

The right side of this inequality is a quadratic polynomial. If we verify that it is nonnegative over $[-1,1)$, then $a(t) \geq h(t)$ over that range (because $F(t) \geq$ $0)$. To check this, we simply check that it is nonnegative at $t= \pm 1$ and has nonpositive leading coefficient. Each of these calculations amounts to checking that a certain explicit linear combination of $a\left(t_{1}\right), \ldots, a\left(t_{8}\right), a^{\prime}\left(t_{1}\right), \ldots, a^{\prime}\left(t_{8}\right), a^{(2)}(-1)$, and $a^{(3)}(-1)$ is nonnegative. We prove it using the method from the previous subsection, with $\ell=36$.

7.3. Proof of uniqueness. Finally, we must deal with the issue of uniqueness when the potential function $f$ is strictly completely monotonic (so $a$ is strictly absolutely monotonic). In that case, by Proposition 2.2 ,

$$
\frac{a(t)-\tilde{h}(t)}{F(t)}
$$


is strictly absolutely monotonic on $(-1,1)$, which implies that

$$
\frac{a(t)-\tilde{h}(t)}{F(t)}>q(t)
$$

for $t \in(-1,1)$. Thus, $a(t)-h(t)$ has roots only at $t=t_{i}$ for some $i$. Uniqueness follows, because the vertices of the regular 600 -cell are the only $(4,120,(1+\sqrt{5}) / 4)$ spherical code (see [Bö, p. 260] or [BD]).

\section{OTHER COMPACT TWO-POINT HOMOGENEOUS SPACES}

The key property underlying our proofs is the two-point homogeneity of the sphere. Recall that a metric space is two-point homogeneous if for each $r>0$, its isometry group acts transitively on ordered pairs of points at distance $r$. In this section we generalize Theorem 1.2 to all compact, connected two-point homogeneous spaces. This generalization is parallel to the general setting for linear programming bounds in [CS, Lev2, Lev3]. It seems plausible that our results also generalize to the case of discrete two-point homogeneous spaces (see Chapter 9 of [CS] for examples), but we have not investigated that possibility.

Let $X$ be a compact, connected two-point homogeneous space with more than one point. Wang proved in Wa that, up to changing to an equivalent metric (i.e., applying an invertible function to the metric), $X$ is one of the following possibilities: a sphere, a real projective space, a complex projective space, a quaternionic projective space, or the octonionic projective plane, each with its standard metric. In other words, we use the round metric on the sphere, the Fubini-Study metric on real, complex, or quaternionic projective space, and the $F_{4}$-invariant metric on $\mathbb{O P}^{2}$ (up to scaling, they are the unique two-point homogeneous Riemannian metrics on these spaces).

The octonionic projective plane $\mathbb{O P}^{2}$ may be the least familiar of these possibilities; it cannot be defined as $\mathbb{O}^{3} \backslash\{0\}$ modulo the action of $\mathbb{O}^{*}$ because of the nonassociativity of the octonions $\left(\mathbb{O}^{*}\right.$ is not a group and does not act on $\left.\mathbb{O}^{3}\right)$. However, it can be defined using the exceptional Jordan algebra or as $F_{4} / \operatorname{Spin}(9)$. There is no such space as $\mathbb{O P}^{3}$, because $\mathbb{O P}^{2}$ does not satisfy the Desargues theorem. See Ba] for more details on octonionic projective geometry.

Normalize the metric $d$ on $X$ (the geodesic distance with respect to the Riemannian metric) so that the greatest distance between points is $\pi$. Just as we use inner products to measure distances between points on spheres, it is natural to use $\cos d(x, y)$ instead of $d(x, y)$. In $\mathbb{R} \mathbb{P}^{n}, \mathbb{C P}^{n}$, or $\mathbb{H} \mathbb{P}^{n}$ this function is easy to calculate: if we represent $x$ and $y$ by unit vectors in $\mathbb{R}^{n+1}, \mathbb{C}^{n+1}$, or $\mathbb{H}^{n+1}$, respectively, then $\cos d(x, y)=2|\langle x, y\rangle|^{2}-1$, where the brackets denote the standard Hermitian form over $\mathbb{R}, \mathbb{C}$, or $\mathbb{H}$.

Spheres are $n$-point homogeneous for every $n$, but the other two-point homogeneous spaces are not even three-point homogeneous. One consequence is that configurations in projective space can frequently be deformed without changing the distances between points. For example, for $\tau \in \mathbb{C}$ with $|\tau|=(1+\sqrt{5}) / 2$, consider the configuration $\mathcal{C}_{\tau}$ consisting of the six points in $\mathbb{C P}^{2}$ with homogeneous coordinates $[\tau, 1,0],[\tau,-1,0],[1,0, \tau],[-1,0, \tau],[0, \tau, 1]$, and $[0, \tau,-1]$. When $\tau=(1+\sqrt{5}) / 2$, they are the images in $\mathbb{R P}^{2} \subset \mathbb{C P}^{2}$ of the vertices of a regular icosahedron in $\mathbb{R}^{3}$. The cosine of the distance between any two distinct points is $-3 / 5$, so the points form a regular simplex embedded in $\mathbb{C P}^{2}$. It will follow from Theorem 8.2 that 
each $\mathcal{C}_{\tau}$ is a universally optimal six-point code in $\mathbb{C P}^{2}$. Lemma 8.1 shows that these configurations form a one-dimensional family. By contrast, positive-dimensional families of sharp configurations do not exist in spheres.

Lemma 8.1. For $|\tau|=\left|\tau^{\prime}\right|=(1+\sqrt{5}) / 2$, there is an isometry of $\mathbb{C P}^{2}$ mapping $C_{\tau}$ to $C_{\tau^{\prime}}$ if and only if $\tau / \tau^{\prime}$ or $\tau / \bar{\tau}^{\prime}$ is a sixth root of unity.

Proof. The isometry group of $\mathbb{C P}^{2}$ is generated by the action of $U(3)$ on lines in $\mathbb{C}^{3}$ together with complex conjugation. Complex conjugation sends $C_{\tau}$ to $C_{\bar{\tau}}$, the diagonal unitary transformation that multiplies the $j$-th coordinate by $e^{2 \pi i j / 3}$ sends $C_{\tau}$ to $C_{\tau e^{-2 \pi i / 3}}$, and the identity map sends $C_{\tau}$ to $C_{-\tau}$. In the other direction, if $x, y$, and $z$ are unit vectors in the six lines in $\mathbb{C}^{3}$ corresponding to the points of $\mathcal{C}_{\tau}$, then

$$
\langle x, y\rangle\langle y, z\rangle\langle z, x\rangle \in\left\{1,1 / 5, \pm 1 /(5 \sqrt{5}), \pm \tau^{3} /\left(|\tau|^{2}+1\right)^{3}, \pm \bar{\tau}^{3} /\left(|\tau|^{2}+1\right)^{3}\right\},
$$

and each of these values occurs (note that the triple product $\langle x, y\rangle\langle y, z\rangle\langle z, x\rangle$ is independent of which unit vector is chosen in each line). Because $|\tau|^{3} /\left(|\tau|^{2}+1\right)^{3}=$ $1 /(5 \sqrt{5})$, the isometry class of $\mathcal{C}_{\tau}$ determines the set $\left\{ \pm 1, \pm \tau^{3}, \pm \bar{\tau}^{3}\right\}$, and that proves the necessity of the condition in the lemma statement.

The potential functions we will consider are the absolutely monotonic functions of $\cos d(x, y)$. As in the case of the sphere, these functions can also be understood in terms of another metric, namely the chordal distance $d_{c}(x, y)=\sin (d(x, y) / 2)$ introduced for Grassmannians in [CHS (i.e., $\sqrt{1-|\langle x, y\rangle|^{2}}$ when $x$ and $y$ are represented by unit vectors as above). For motivation, recall that the Euclidean distance between two points separated by an angle of $\theta$ on the unit sphere is $2 \sin (\theta / 2)$. For projective spaces, the chordal distance is most naturally expressed in terms of the orthogonal projection operators $\Pi_{x}$ and $\Pi_{y}$ onto the lines $x$ and $y$. Then

$$
d_{c}(x, y)=\frac{\left|\Pi_{x}-\Pi_{y}\right|_{F}}{\sqrt{2}},
$$

where $|\cdot|_{F}$ is the Frobenius norm, defined by

$$
|A|_{F}^{2}=\sum_{i, j}\left|A_{i, j}\right|^{2}=\operatorname{tr} \bar{A}^{t} A
$$

Absolutely monotonic functions of $\cos d(x, y)$ are the same as completely monotonic functions of squared chordal distance, just as in the case of the unit sphere.

Let $G$ be the isometry group of $X$. Then one can decompose $L^{2}(X)$ as

$$
L^{2}(X)=\widehat{\bigoplus_{\ell \geq 0}} V_{\ell},
$$

where $V_{0}, V_{1}, \ldots$ are certain finite-dimensional irreducible representations of $G$. This decomposition is unique because the summands are pairwise nonisomorphic. For each $\ell$, one can define a positive-definite kernel $K_{\ell}$ on $X$ as in the case of the sphere, and one can define a function $C_{\ell}:[-1,1] \rightarrow \mathbb{R}$ such that

$$
K_{\ell}(x, y)=C_{\ell}(\cos d(x, y)) \text {. }
$$

We index the representations $V_{\ell}$ so that $C_{\ell}$ is a polynomial of degree $\ell$. One can compute these polynomials explicitly (see for example [Gan, p. 178]). For projective spaces, the result is that $C_{\ell}$ is a positive constant times the Jacobi polynomial 
$P_{\ell}^{(\alpha, \beta)}$, where $\alpha=n / 2-1, n$ is the dimension of $X$ as a real manifold, and $\beta$ is $-1 / 2,0,1$, or 3 according to whether one is working over $\mathbb{R}, \mathbb{C}, \mathbb{H}$, or $\mathbb{O}$, respectively (i.e., $\beta=\left(\operatorname{dim}_{\mathbb{R}} A\right) / 2-1$ for the algebra $\left.A\right)$. We define a positive-definite polynomial to be a nonnegative linear combination of $C_{0}, C_{1}, \ldots$

An $M$-design in $X$ is a finite subset $\mathcal{C} \subset X$ such that for $f \in V_{i}$ with $1 \leq i \leq M$,

$$
\sum_{x \in \mathcal{C}} f(x)=0
$$

As in the case of spheres, being an $M$-design is also characterized by the requirement that

$$
\sum_{x, y \in \mathcal{C}} C_{i}(\cos d(x, y))=0
$$

for $1 \leq i \leq M$. For example, to verify that the configurations $\mathcal{C}_{\tau}$ constructed above are 1-designs in $\mathbb{C P}^{2}$, one need only check that $6 C_{1}(1)+30 C_{1}(-3 / 5)=0$.

We say that a finite subset $\mathcal{C} \subset X$ is a sharp configuration if it is an $M$-design, $m$ distances occur between distinct points in $\mathcal{C}$, and $M \geq 2 m-1-\delta$, where $\delta=1$ if there are two antipodal points in $\mathcal{C}$ and $\delta=0$ otherwise. By a pair of "antipodal points" we mean points separated by the greatest possible distance in $X$; this condition is more subtle in projective spaces than in spheres, because it is no longer true that each point has a unique antipode.

This definition of a sharp configuration agrees with the previous one in the case of spheres. Here we merely require an antipodal sharp configuration to be a $(2 m-2)$ design, but in the case of spheres every antipodal $(2 m-2)$-design is automatically a $(2 m-1)$-design: by Theorem 7.4 of [DGS, every point in such a design has an antipode if one does, and that suffices to make every odd-degree homogeneous polynomial sum to zero. On the other hand, some antipodal sharp configurations in projective space are only $(2 m-2)$-designs. For example, the $D_{4}, E_{6}$, and $E_{7}$ root systems give antipodal sharp configurations of 12,36 , and 63 points in $\mathbb{R P}^{3}$, $\mathbb{R} \mathbb{P}^{5}$, and $\mathbb{R P}^{6}$, respectively. For these codes $m=2$ (the cosines of the distances are -1 and $-1 / 2$ ), and they are 2 -designs but not 3 -designs. By contrast, $E_{8}$ yields a 3 -design in $\mathbb{R} \mathbb{P}^{7}$.

The following theorem generalizes Theorem 1.2 and the results of Levenshtein from [Lev2] (namely, that all sharp configurations are optimal codes):

Theorem 8.2. Let $f:[-1,1) \rightarrow \mathbb{R}$ be absolutely monotonic, and let $\mathcal{C} \subset X$ be a sharp configuration. If $\mathcal{C}^{\prime} \subset X$ is any subset satisfying $\left|\mathcal{C}^{\prime}\right|=|\mathcal{C}|$, then

$$
\sum_{x, y \in \mathcal{C}^{\prime}, x \neq y} f(\cos d(x, y)) \geq \sum_{x, y \in \mathcal{C}, x \neq y} f(\cos d(x, y)) .
$$

If $f$ is strictly absolutely monotonic, then equality implies that $\mathcal{C}^{\prime}$ is a sharp configuration and that the same distances occur between points in $\mathcal{C}$ and $\mathcal{C}^{\prime}$.

Naturally, we call

$$
\sum_{x, y \in \mathcal{C}, x \neq y} f(\cos d(x, y))
$$

the $f$-potential energy of $\mathcal{C}$. Strictly speaking, this definition conflicts with our earlier definition for the special case $X=S^{n-1}$, because previously we used functions of squared Euclidean distance as our potential functions. However, that should not cause any confusion: the Euclidean distance is twice the chordal distance, so this 
change simply amounts to the difference between using the squared chordal distance and the cosine of the geodesic distance. In the terminology of Section 6 , here we are dealing with the absolutely monotonic function $a$ instead of the completely monotonic potential function, and thus our previous framework is equivalent to the current one in the case of $S^{n-1}$.

The proof of Theorem 8.2 is parallel to the proof for spheres, so we merely sketch it here, with particular attention to arguments that require modification in projective space.

The linear programming bounds generalize in a straightforward manner, with essentially the same proof:

Proposition 8.3. Let $f:[-1,1) \rightarrow \mathbb{R}$ be any function. Suppose $h:[-1,1] \rightarrow \mathbb{R}$ is a polynomial such that $h(t) \leq f(t)$ for all $t \in[-1,1)$, and suppose there are nonnegative coefficients $\alpha_{0}, \ldots, \alpha_{d}$ such that $h$ has the expansion

$$
h(t)=\sum_{i=0}^{d} \alpha_{i} C_{i}(t) .
$$

Then every set of $N$ points in $X$ has $f$-potential energy at least

$$
N^{2} \alpha_{0}-N h(1) \text {. }
$$

We apply this proposition in the same way as before. Suppose first that $\mathcal{C}$ is a $(2 m-1)$-design, with $m$ distances between distinct points in $\mathcal{C}$. Let $t_{1}<\cdots<t_{m}$ be the cosines of those distances. As before, we construct the Hermite interpolating polynomial $h$ of degree $2 m-1$ that agrees with $f$ to order 2 at each of $t_{1}, \ldots, t_{m}$. The proof that $h(t) \leq f(t)$ for all $t \in[-1,1)$ is the same as in Lemma 6.1.

Define

$$
F(t)=\prod_{i=1}^{m}\left(t-t_{i}\right) .
$$

The same proof as in Lemma 6.2 shows that $F$ is positive definite, and we apply an analogue of Lemma 6.3 to express $g$ in terms of a related family of orthogonal polynomials as $p_{m}+\alpha p_{m-1}$. To complete the proof that $h$ is positive definite, we use the fact that the polynomials in that family are themselves positive definite, together with Theorem [3.1] to establish conductivity. Everything is completely parallel to the spherical case. Finally, because $\mathcal{C}$ is a $(2 m-1)$-design and $\operatorname{deg}(h)=$ $2 m-1$, the lower bound for potential energy must be sharp. When $f$ is strictly absolutely monotonic, the uniqueness argument is the same as before.

When $t_{1}=-1$ and $\mathcal{C}$ is a $(2 m-2)$-design but not a $(2 m-1)$-design, one must instead use first-order Hermite interpolation at $t=-1$, which complicates the proof. We can assume that $m>1$, since if $m=1$, then every pair of points in $\mathcal{C}$ is antipodal and Theorem 8.2 visibly holds.

If we define

$$
F(t)=\prod_{i=2}^{m}\left(t-t_{i}\right),
$$

then the heart of the proof is the verification that $(t+1) F(t)^{2}$ is strictly conductive, and everything else works exactly the same as before. Let $q_{0}, q_{1}, \ldots$ be the monic orthogonal polynomials with respect to $\left(1-t^{2}\right) d \mu(t)$, where $\mu$ is the measure on $[-1,1]$ with respect to which the polynomials $C_{\ell}$ are orthogonal. (Recall that they 
are certain Jacobi polynomials $P_{\ell}^{(\alpha, \beta)}$. Then $d \mu(t)=(1-t)^{\alpha}(1+t)^{\beta} d t$.) The argument used to prove Lemma 6.3 shows that $F=q_{m-1}+\alpha q_{m-2}$ for some $\alpha$.

The most subtle part of the proof of Theorem 8.2 is the proof that $\alpha>0$. (If $\mathcal{C}$ were a $(2 m-1)$-design, then $\alpha$ would vanish.) We start with a crucial observation about the polynomials $q_{i}$ :

Lemma 8.4. For each $i$, the polynomial $(t+1) q_{i}(t)$ is strictly positive definite.

Proof. This polynomial is orthogonal to all polynomials of degree less than $i$ with respect to $(1-t) d \mu(t)$, so for some constant $c$ it equals $p_{i+1}(t)+c p_{i}(t)$ in terms of the monic orthogonal polynomials for this measure. Setting $t=-1$ shows that $c \geq 0$, because each $p_{j}$ has all its roots in $(-1,1)$ and hence $p_{j}(-1)$ has sign $(-1)^{j}$. As before, it follows from Lemma 3.3 and Proposition 3.2 that each $p_{j}$ is strictly positive definite.

Lemma 8.5. Let $f(t)$ be a monic polynomial, with 0 -th coefficient $f_{0}$ in terms of $C_{0}(t), C_{1}(t), \ldots$

(1) If $\operatorname{deg}(f) \leq 2 m-2$ and $f\left(t_{i}\right)=0$ for $1 \leq i \leq m$, then $f_{0}|\mathcal{C}|=f(1)$.

(2) If $\operatorname{deg}(f)=2 m-1$ and $f\left(t_{i}\right)=0$ for $1 \leq i \leq m$, then $f_{0}|\mathcal{C}|<f(1)$.

(3) If $\operatorname{deg}(f) \leq 2 m-2$ and $f(t) \geq 0$ for $-1 \leq t \leq 1$, then $f_{0}|\mathcal{C}| \geq f(1)$.

Proof. Write

$$
f(t)=\sum_{i=0}^{\operatorname{deg}(f)} f_{i} C_{i}(t)
$$

In cases (1) and (2),

$$
\sum_{x, y \in \mathcal{C}} f(\cos d(x, y))=|\mathcal{C}| f(1)
$$

while in case $(3)$,

$$
\sum_{x, y \in \mathcal{C}} f(\cos d(x, y)) \geq|\mathcal{C}| f(1)
$$

On the other hand,

$$
\sum_{x, y \in \mathcal{C}} f(\cos d(x, y))=\sum_{i=0}^{\operatorname{deg}(f)} f_{i} \sum_{x, y \in \mathcal{C}} C_{i}(\cos d(x, y)) .
$$

The $i=0$ term yields $f_{0}|\mathcal{C}|^{2}$, while all terms with $1 \leq i \leq 2 m-2$ vanish because $\mathcal{C}$ is a $(2 m-2)$-design. In case (1) that yields $f_{0}|\mathcal{C}|=f(1)$ and in case (3) it yields $f_{0}|\mathcal{C}| \geq f(1)$. Finally, in case (2) we have $f_{2 m-1}>0$ because $f$ is monic, so the $i=2 m-1$ term is positive (because $\mathcal{C}$ is not a $(2 m-1)$-design) and hence $f_{0}|\mathcal{C}|<f(1)$.

Lemma 8.6. If $f$ is a polynomial with $\operatorname{deg}(f) \leq i$, then $(t+1) f(t) q_{i}(t)$ has 0-th coefficient equal to $f(1)$ times that of $(t+1) q_{i}(t)$ (in terms of $\left.C_{0}(t), C_{1}(t), \ldots\right)$.

Proof. The 0 -th coefficient of a polynomial $p(t)$ equals

$$
\frac{\int p(t) d \mu(t)}{\int d \mu(t)} \text {. }
$$

Divide $f(t)$ by $t-1$ to yield $f(t)=g(t)(t-1)+f(1)$, with $\operatorname{deg}(g)<i$. Then

$$
\int(t+1) f(t) q_{i}(t) d \mu(t)=\int g(t) q_{i}(t)\left(1-t^{2}\right) d \mu(t)+f(1) \int(1+t) q_{i}(t) d \mu(t) .
$$


Because $q_{i}$ is orthogonal to all polynomials of degree less than $i$ with respect to $\left(1-t^{2}\right) d \mu(t)$

$$
\int(t+1) f(t) q_{i}(t) d \mu(t)=f(1) \int(1+t) q_{i}(t) d \mu(t),
$$

which completes the proof.

Let $\beta_{i}$ denote the 0 -th coefficient of $(t+1) q_{i}(t)$ in terms of $C_{0}(t), C_{1}(t), \ldots$ Taking $f(t)=(t+1) F(t)$ in case (1) of Lemma 8.5, we find that

$$
\left(\beta_{m-1}+\beta_{m-2} \alpha\right)|\mathcal{C}|=2 q_{m-1}(1)+2 q_{m-2}(1) \alpha .
$$

For an upper bound on $|\mathcal{C}|$, take $f(t)=(t+1) F(t) q_{m-1}(t)$ in case (2). By Lemma 8.6

$$
|\mathcal{C}|<\frac{f(1)}{F(1) \beta_{m-1}}=\frac{2 q_{m-1}(1)}{\beta_{m-1}}
$$

(because $F(1) \beta_{m-1}>0$ ). For a lower bound, take $f(t)=(t+1) q_{m-2}(t)^{2}$ in case (3). By Lemma 8.6 .

$$
|\mathcal{C}| \geq \frac{f(1)}{q_{m-2}(1) \beta_{m-2}}=\frac{2 q_{m-2}(1)}{\beta_{m-2}}
$$

(because $q_{m-2}(1) \beta_{m-2}>0$ ). We have shown that

$$
2 q_{m-1}(1)-\beta_{m-1}|\mathcal{C}|>0
$$

and

$$
2 q_{m-2}(1)-\beta_{m-2}|\mathcal{C}| \leq 0,
$$

while

$$
2 q_{m-1}(1)-\beta_{m-1}|\mathcal{C}|+\alpha\left(2 q_{m-2}(1)-\beta_{m-2}|\mathcal{C}|\right)=0 .
$$

It follows that $\alpha>0$.

To prove that $(t+1) F(t)^{2}$ is strictly conductive using Lemma 5.3. we need only show that all its partial products

$$
(t+1) \prod_{i=2}^{j}\left(t-t_{i}\right)^{2}
$$

and

$$
(t+1)\left(t-t_{j+1}\right) \prod_{i=2}^{j}\left(t-t_{i}\right)^{2}
$$

are strictly positive definite. That follows from Theorem 3.1, Lemma 8.4, and the fact that the set of nonnegative linear combinations of $q_{0}, q_{1}, q_{2}, \ldots$ is closed under taking products (see Gas for the most general case or Theorem 5.2 in $\mathrm{As}$, p. 44] for a simpler proof that applies to these orthogonal polynomials). Once the conductivity of $(t+1) F(t)^{2}$ has been established, the rest of the proof of Theorem 8.2 is essentially identical to that of Theorem 1.2 , 


\section{The Euclidean CASE}

One can generalize Proposition 4.1 to apply to configurations in Euclidean space. Here we outline this approach, which leads to an optimization problem reminiscent of the Beurling-Selberg extremal functions (see $\mathrm{V}]$ ). We deal only with the case of periodic, discrete point configurations (unions of finitely many translates of a lattice), to avoid worries about how potential energy is defined in pathological cases.

Define the density of a periodic arrangement to be the number of points per unit volume in space. If the arrangement consists of the $N$ translates $\Lambda+v_{1}, \ldots, \Lambda+v_{N}$ of a lattice $\Lambda \in \mathbb{R}^{n}$, with $v_{j}-v_{k} \notin \Lambda$ for $j \neq k$, then the density is $N / \operatorname{vol}\left(\mathbb{R}^{n} / \Lambda\right)$. Given a potential function $f:(0, \infty) \rightarrow[0, \infty)$, define the potential energy of the packing to be

$$
\frac{1}{N} \sum_{1 \leq j, k \leq N} \sum_{x \in \Lambda, x+v_{j}-v_{k} \neq 0} f\left(\left|x+v_{j}-v_{k}\right|^{2}\right) .
$$

Of course summing over all pairs of points in the packing would give a divergent sum (even this sum may diverge, in which case we say the potential energy is infinite). The potential energy is the average over all points in the configuration of the sum over all distances to other points. In particular, the same periodic arrangement can be written in terms of different $\Lambda$ and $v_{1}, \ldots, v_{N}$, but the potential energy is independent of this choice.

For a different point of view, imagine placing finitely many points $v_{1}, \ldots, v_{N}$ on the flat torus $\mathbb{R}^{n} / \Lambda$. The energy of a point is given by a sum over all geodesics connecting it to the other points or to itself, and the energy of the configuration is the average of this quantity over all the points.

Let $B_{R}(0)$ denote the ball of radius $R$ about 0 . Given a configuration $\mathcal{C} \subset \mathbb{R}^{n}$, let

$$
\mathcal{C}_{R}=\mathcal{C} \cap B_{R}(0) .
$$

Lemma 9.1. Let $\mathcal{C}$ be a periodic point configuration in $\mathbb{R}^{n}$. If the potential energy of $\mathcal{C}$ is finite, then it equals

$$
\lim _{R \rightarrow \infty} \frac{1}{\left|\mathcal{C}_{R}\right|} \sum_{x, y \in \mathcal{C}_{R}, x \neq y} f\left(|x-y|^{2}\right) .
$$

The same holds even without the assumption that $f$ is nonnegative, as long as $f(x)=O\left((1+|x|)^{-n / 2-\delta}\right)$ for some $\delta>0$.

Proof. Suppose $\mathcal{C}$ is the disjoint union of $\Lambda+v_{1}, \ldots, \Lambda+v_{N}$ as above. Say a point in $\mathcal{C}$ has type $j$ if it is in $\Lambda+v_{j}$. With each point $x \in \mathcal{C}$ we associate the sum

$$
\sum_{y \in \mathcal{C}, y \neq x} f\left(|x-y|^{2}\right) .
$$

This sum depends only on the type of $x$, and the definition of the $f$-potential energy of $\mathcal{C}$ equals the average over all types of this quantity.

All these sums must converge, because of the bounds on $f$ if $f$ is not assumed nonnegative or else because of the hypothesis that the potential energy is finite. Let $\varepsilon>0$ be small, and choose $K$ such that for $x$ of each type,

$$
\sum_{y \in \mathcal{C},|y-x|>K}\left|f\left(|x-y|^{2}\right)\right| \leq \varepsilon .
$$


In particular, summing in (9.1) only over $y$ such that $|x-y| \leq K$ yields a partial sum within $\varepsilon$ of the total sum.

In the sum

$$
\sum_{x, y \in \mathcal{C}_{R}, x \neq y} f\left(|x-y|^{2}\right),
$$

we can focus our attention on the points $x \in B_{R-K}(0)$, since the number in $B_{R}(0) \backslash$ $B_{R-K}(0)$ is negligible compared to the number in $B_{R-K}(0)$ (on the order of $R^{n-1}$ rather than $R^{n}$ as $\left.R \rightarrow \infty\right)$. Each point contributes a bounded amount to the sum, so omitting the points in $B_{R}(0) \backslash B_{R-K}(0)$ changes the sum by $O\left(R^{n-1}\right)$.

For each $x \in \mathcal{C}_{R-K}$,

$$
\sum_{y \in \mathcal{C}_{R}, y \neq x} f\left(|x-y|^{2}\right)
$$

is within $\varepsilon$ of

$$
\sum_{y \in \mathcal{C}, y \neq x} f\left(|x-y|^{2}\right) .
$$

The numbers of points of each type in $\mathcal{C}_{R-K}$ are equal, up to a factor of $1+O(1 / R)$. Thus, for $R$ large,

$$
\frac{1}{\left|\mathcal{C}_{R}\right|} \sum_{x, y \in \mathcal{C}_{R}, x \neq y} f\left(|x-y|^{2}\right)
$$

is $O(1 / R)$ plus a quantity within $\varepsilon$ of the $f$-potential energy of $\mathcal{C}$. Choosing $\varepsilon$ arbitrarily small and $R$ accordingly large completes the proof.

The Euclidean analogue of Proposition 4.1 is stated in terms of the Fourier transform on $\mathbb{R}^{n}$. We normalize the Fourier transform of an $L^{1}$ function $h: \mathbb{R}^{n} \rightarrow \mathbb{R}$ by

$$
\widehat{h}(t)=\int_{\mathbb{R}^{n}} h(x) e^{2 \pi i\langle x, t\rangle} d x .
$$

Lemma 9.2. Let $\mathcal{C}$ be a periodic point configuration in $\mathbb{R}^{n}$, with density $\delta$. If $\varepsilon>0$ is sufficiently small (depending on $\mathcal{C}$ ), then

$$
\lim _{R \rightarrow \infty} \int_{B_{\varepsilon}(0)} \frac{\left|\sum_{x \in \mathcal{C}_{R}} e^{2 \pi i\langle x, t\rangle}\right|^{2}}{\left|\mathcal{C}_{R}\right|} d t=\delta .
$$

Proof. We will prove that for $\varepsilon>0$ sufficiently small, if $g$ is any radial, smooth function supported in $B_{\varepsilon}(0)$, then

$$
\lim _{R \rightarrow \infty} \int_{\mathbb{R}^{n}} \frac{\left|\sum_{x \in \mathcal{C}_{R}} e^{2 \pi i\langle x, t\rangle}\right|^{2}}{\left|\mathcal{C}_{R}\right|} g(t) d t=\delta g(0) .
$$

Because the characteristic function of a small ball can be bounded above and below by such functions, that will suffice to prove the lemma.

We expand the numerator of the integrand via

$$
\left|\sum_{x \in \mathcal{C}_{R}} e^{2 \pi i\langle x, t\rangle}\right|^{2}=\sum_{x, y \in \mathcal{C}_{R}} e^{2 \pi i\langle x-y, t\rangle} .
$$

It follows that

$$
\int_{\mathbb{R}^{n}} \frac{\left|\sum_{x \in \mathcal{C}_{R}} e^{2 \pi i\langle x, t\rangle}\right|^{2}}{\left|\mathcal{C}_{R}\right|} g(t) d t=\frac{1}{\left|\mathcal{C}_{R}\right|} \sum_{x, y \in \mathcal{C}_{R}} \widehat{g}(x-y) .
$$


Let $\mathcal{C}$ be the disjoint union of translates $\Lambda+v_{1}, \ldots, \Lambda+v_{N}$ of a lattice $\Lambda$. It follows from Lemma 9.1 that

$$
\lim _{R \rightarrow \infty} \frac{1}{\left|\mathcal{C}_{R}\right|} \sum_{x, y \in \mathcal{C}_{R}} \widehat{g}(x-y)=\frac{1}{N} \sum_{1 \leq j, k \leq N} \sum_{z \in \Lambda} \widehat{g}\left(z+v_{j}-v_{k}\right) .
$$

(This quantity is not quite the same as that considered in Lemma 9.1 because we allow $x=y$ or $z+v_{j}-v_{k}=0$ in the sum, but the extra terms amount to $\widehat{g}(0)$ on each side.)

Recall the Poisson summation formula, which states that for every Schwartz function $h: \mathbb{R}^{n} \rightarrow \mathbb{R}$ and every $v \in \mathbb{R}^{n}$,

$$
\sum_{z \in \Lambda} h(z+v)=\frac{1}{\operatorname{vol}\left(\mathbb{R}^{n} / \Lambda\right)} \sum_{t \in \Lambda^{*}} \widehat{h}(t) e^{-2 \pi i\langle v, t\rangle},
$$

where

$$
\Lambda^{*}=\left\{t \in \mathbb{R}^{n}:\langle t, z\rangle \in \mathbb{Z} \text { for all } z \in \Lambda\right\}
$$

is the dual lattice. (See Appendix A of [CK2] for the standard proof.) Taking $h=\widehat{g}$ yields

$$
\frac{1}{N} \sum_{1 \leq j, k \leq N} \sum_{z \in \Lambda} \widehat{g}\left(z+v_{j}-v_{k}\right)=\frac{1}{N \operatorname{vol}\left(\mathbb{R}^{n} / \Lambda\right)} \sum_{t \in \Lambda^{*}} g(t)\left|\sum_{1 \leq j \leq N} e^{2 \pi i\left\langle v_{j}, t\right\rangle}\right|^{2} .
$$

If the support of $g$ is sufficiently small, then only the $t=0$ term contributes to the right side, and it equals $\delta g(0)$ because $N / \operatorname{vol}\left(\mathbb{R}^{n} / \Lambda\right)=\delta$.

Proposition 9.3. Let $f:(0, \infty) \rightarrow[0, \infty)$ be any function. Suppose $h: \mathbb{R}^{n} \rightarrow \mathbb{R}$ satisfies $h(x) \leq f\left(|x|^{2}\right)$ for all $x \in \mathbb{R}^{n} \backslash\{0\}$ and is the Fourier transform of a function $g \in L^{1}\left(\mathbb{R}^{n}\right)$ such that $g(t) \geq 0$ for all $t \in \mathbb{R}^{n}$. Then every periodic configuration in $\mathbb{R}^{n}$ with density $\delta$ has $f$-potential energy at least

$$
\delta\left(\liminf _{t \rightarrow 0} g(t)\right)-h(0) .
$$

Without loss of generality, one can assume that $g$ and $h$ are radial functions (replace $g$ with the average of its rotations about the origin). For simplicity of notation, we typically write $g=\widehat{h}$. That is justified because $g$ is in fact the Fourier transform of $h$ as a tempered distribution (when these functions are radial; of course in general $g(t)=\widehat{h}(-t)$ ), but note that we have not assumed that $h$ is integrable and thus it is not clear that

$$
\int_{\mathbb{R}^{n}} h(x) e^{2 \pi i\langle x, t\rangle} d x
$$

converges. By contrast, $h(x)$ does equal

$$
\int_{\mathbb{R}^{n}} \widehat{h}(t) e^{2 \pi i\langle x, t\rangle} d t
$$

Proof. Define

$$
\ell=\liminf _{t \rightarrow 0} g(t) .
$$

Let $\varepsilon>0$, and choose $\eta>0$ such that $g(t) \geq \ell-\varepsilon$ whenever $|t| \leq \eta$. 
Let $\mathcal{C}$ be a periodic configuration with density $\delta$. By Lemma 9.1, the $f$-potential energy is

$$
\lim _{R \rightarrow \infty} \frac{1}{\left|\mathcal{C}_{R}\right|} \sum_{x, y \in \mathcal{C}_{R}, x \neq y} f\left(|x-y|^{2}\right) .
$$

Because $h(x) \leq f\left(|x|^{2}\right)$ for all $x \neq 0$,

$$
\frac{1}{\left|\mathcal{C}_{R}\right|} \sum_{x, y \in \mathcal{C}_{R}, x \neq y} f\left(|x-y|^{2}\right) \geq-h(0)+\frac{1}{\left|\mathcal{C}_{R}\right|} \sum_{x, y \in \mathcal{C}_{R}} h(x-y) .
$$

On the other hand,

$$
\begin{aligned}
\frac{1}{\left|\mathcal{C}_{R}\right|} \sum_{x, y \in \mathcal{C}_{R}} h(x-y) & =\frac{1}{\left|\mathcal{C}_{R}\right|} \sum_{x, y \in \mathcal{C}_{R}} \int_{\mathbb{R}^{n}} g(t) e^{2 \pi i\langle x-y, t\rangle} d t \\
& =\int_{\mathbb{R}^{n}} g(t) \frac{\left|\sum_{x \in \mathcal{C}_{R}} e^{2 \pi i\langle x, t\rangle}\right|^{2}}{\left|\mathcal{C}_{R}\right|} d t \\
& \geq \int_{B_{\eta}(0)}(\ell-\varepsilon) \frac{\left|\sum_{x \in \mathcal{C}_{R}} e^{2 \pi i\langle x, t\rangle}\right|^{2}}{\left|\mathcal{C}_{R}\right|} d t .
\end{aligned}
$$

In the limit as $R \rightarrow \infty$, Lemma 9.2 implies that the potential energy is at least

$$
-h(0)+\delta(\ell-\varepsilon),
$$

if $\eta$ is sufficiently small. Because $\varepsilon$ can be taken to be arbitrarily small, the desired lower bound follows.

This proposition is closely analogous to the linear programming bounds for sphere packing introduced in [CE] (see also [Co, CK1, CK2]). The proof given here is somewhat different, because we have arranged it to minimize the hypotheses on $h$. If one is willing to assume stronger hypotheses, namely bounds on the decay rates of $h$ and $\widehat{h}$, then one can give a proof closer to that in $\mathrm{CE}$ by moving the Poisson summation from the proof of Lemma 9.2 into that of Proposition 9.3 .

Analogously to the case of Proposition 4.1, one can derive the bounds from $\mathrm{CE}$ (with weaker hypotheses than in that paper) by setting

$$
f(r)= \begin{cases}\infty & \text { for } r<1, \text { and } \\ 0 & \text { otherwise }\end{cases}
$$

Then one concludes that the sphere centers in a packing with balls of radius $1 / 2$ must have density at most $h(0) / \widehat{h}(0)$ (assuming $\widehat{h}$ is continuous at 0 ).

Let $\Lambda_{2}, \Lambda_{8}$, and $\Lambda_{24}$ denote the hexagonal lattice in $\mathbb{R}^{2}$, the $E_{8}$ root lattice in $\mathbb{R}^{8}$, and the Leech lattice in $\mathbb{R}^{24}$, respectively.

Conjecture 9.4. Let $n \in\{2,8,24\}$, and let $f:(0, \infty) \rightarrow \mathbb{R}$ be completely monotonic and satisfy $f(x)=O\left(|x|^{-n / 2-\varepsilon}\right)$ as $|x| \rightarrow \infty$ for some $\varepsilon>0$. Then there exists a function $h$ that satisfies the hypotheses of Proposition 9.3 and proves that $\Lambda_{n}$ has the least $f$-potential energy of any periodic configuration in $\mathbb{R}^{n}$ with its density.

One consequence of this conjecture is that these lattices are universally optimal configurations in Euclidean space. That appears to be a rare property, which provably fails for lattices in dimensions $3,5,6$, and 7 . The unique optimal lattices in those dimensions are the $A_{3}, D_{5}, E_{6}$, and $E_{7}$ root lattices (see $\underline{\mathrm{Mar}}$ ). Each 
of these lattices has higher energy than its dual lattice for the potential function $f(x)=e^{-x}$, when both lattices are rescaled to have unit density.

Montgomery proved in $[\mathrm{Mo}$ that the hexagonal lattice is universally optimal among all lattices in $\mathbb{R}^{2}$, but that is a weaker assertion than universal optimality among all periodic point configurations. Sarnak and Strömbergsson conjectured in SS that $D_{4}, E_{8}$, and the Leech lattice are universally optimal among lattices and proved that they are local optima. Perhaps one could combine their theorem with the techniques in this paper and [CK1] to prove that $E_{8}$ and $\Lambda_{24}$ are the global minima among lattices in their dimensions.

If true, Conjecture 9.4 implies that $E_{8}$ and the Leech lattice are the densest sphere packings in their dimensions, by using the potential function $f(r)=1 / r^{s}$ with $s$ large. (The density of a sphere packing is the fraction of space covered by the spheres; unfortunately this terminology conflicts with the density of a point configuration. We will refer to these numbers as the "packing density" and "point density" to avoid confusion.) The potential energy of any periodic packing is asymptotic to

$$
\frac{K}{r_{\min }^{2 s}}
$$

as $s \rightarrow \infty$, where $r_{\min }$ is the smallest distance between distinct points and $K$ is the average number of points at distance $r_{\min }$ from a point in the packing (i.e., the average kissing number if one centers balls of radius $r_{\min } / 2$ at the points of the arrangement to form a sphere packing). Universal optimality implies that $r_{\min }$ must be as large as possible for a periodic configuration of point density 1 , which means the packing density is maximized. Thus, Conjecture 9.4 implies that no periodic packing can exceed the packing density of $E_{8}$ or the Leech lattice, and therefore no packing can (see Appendix A of [CE]).

Furthermore, Conjecture 9.4 implies that $E_{8}$ and the Leech lattice are the unique densest periodic packings in $\mathbb{R}^{8}$ and $\mathbb{R}^{24}$. In the notation used above, any equally dense periodic packing must have at least as large an average kissing number $K$, or the potential energy would be lower. Because $E_{8}$ and $\Lambda_{24}$ have the greatest possible kissing numbers in their dimensions and those kissing configurations are unique (see Chapters 13 and 14 of [CS]), the kissing configurations in the hypothetical packing are determined up to orthogonal transformations. However, those orthogonal transformations may be nontrivial, so it is not obvious that the packing is uniquely determined.

Lemma 9.5. For $n=8$ or $n=24$, there is a unique sphere packing in $\mathbb{R}^{n}$ such that each kissing configuration is the $\Lambda_{n}$ kissing configuration.

Proof. Without loss of generality, suppose one sphere is centered at a point in $\Lambda_{n}$ and the spheres tangent to it are centered at the nearest points in $\Lambda_{n}$. We must prove that each sphere is centered at a point of $\Lambda_{n}$. Call a sphere center good if it is in $\Lambda_{n}$ and the same is true for all spheres tangent to it, and call one point a neighbor of another if they are the centers of tangent spheres. By assumption, at least one point is good; call it the origin. We will prove that all neighbors of good points are also good. It follows that all points in the packing are good. To see why, suppose $x$ is not good with $|x|$ minimal. Then $x$ must have a neighbor $y$ with $|y|<|x|$, since otherwise a suitable scalar multiple of $x$ (chosen between $x$ and the origin to match the minimal distance of the packing) could be included with the neighbors of $x$ to increase the kissing number in $\mathbb{R}^{n}$; the reason is that every 
neighbor of $x$ would be on or outside the sphere of radius $|x|$ and hence at least as far from the new point as $x$ is. The smaller neighbor $y$ is good by assumption, which implies that $x$ is good.

It remains to prove that all neighbors of good points are good. Suppose $x$ is good and $y$ is a neighbor of $x$. We must show that there is a unique way to arrange the neighbors of $y$ (namely, as points of $\Lambda_{n}$ ). The positions of the common neighbors of $x$ and $y$ are already determined, because $x$ is good. It would suffice to show that every isometry of $\mathbb{R}^{n}$ that fixes $x$ and $y$ and preserves the set of common neighbors must preserve $\Lambda_{n}$, because any two possible kissing arrangements about $y$ are related by such an isometry. (They are related by some isometry fixing $y$ by the uniqueness of the kissing configuration. Because the symmetry group acts transitively on the neighbors (see Lemma 1.10 in [Eb, p. 25] and Theorem 27 in CS. p. 288]), we may assume that $x$ is fixed as well, and it follows that the set of common neighbors is also preserved.) For $n=8$ we will show that this is true; for $n=24$ it is false, but we will prove a slightly weaker statement that will still suffice.

Without loss of generality, we can translate the origin so that $y=0$. When $n=8$, $x$ and its 56 common neighbors with 0 span $E_{8}$, so every orthogonal transformation that preserves $x$ and the common neighbors clearly preserves $E_{8}$. That completes the proof by showing that the kissing arrangements about two neighbors can only be in one orientation relative to each other.

When $n=24, x$ and its 4600 common neighbors with 0 do not span $\Lambda_{24}$ (their span cannot contain any vector that has an odd inner product with $x$ ). Instead, they span a sublattice $L$ of index 2, as is not hard to check using Figure 4.12 in CS, p. 133] (with $x=(4,-4,0, \ldots, 0) / \sqrt{8})$. This lattice contains all the minimal vectors from $\Lambda_{24}$ that have even inner product with $x$, and it contains twice the minimal vectors from $\Lambda_{24}$ that have inner product \pm 1 with $x$.

The dual lattice

$$
L^{*}=\left\{w \in \mathbb{R}^{n}:\langle w, z\rangle \in \mathbb{Z} \text { for all } z \in L\right\}
$$

contains $L$ (because $L$ is integral), and considering covolumes shows that $\left[L^{*}: L\right]=$ 4. The Leech lattice is contained in $L^{*}$, as is the vector $x / 2$, and they must generate $L^{*}$ because they generate a lattice in which $L$ has index 4 .

Every orthogonal transformation that preserves $x$ and the set of 4600 common neighbors preserves $L$ and hence $L^{*}$. That means the kissing configuration of our packing at 0 must be contained among the vectors of norm 4 in $L^{*}$, because there exists such an orthogonal transformation that yields the Leech kissing configuration, which is contained in $L^{*}$. Besides the minimal vectors of $\Lambda_{24}$, the only other possibilities are the vectors of the form $\pm(x / 2-z)$ where $z$ is a minimal vector of $\Lambda_{24}$ satisfying $\langle x, z\rangle=1$. (Any vector in $L^{*}$ but not $\Lambda_{24}$ must be of the form $x / 2-w$ with $w \in \Lambda_{24}$. A short calculation shows that if $|x / 2-w|^{2}=4$, then either $w=z$ with $|z|^{2}=4$ and $\langle x, z\rangle=1$ or $w=x-z$ for such a $z$.)

The kissing configuration must contain all the minimal vectors of $L$, namely the minimal vectors of $\Lambda_{24}$ that have even inner product with $x$, because $L$ is preserved under the transformation to the Leech kissing configuration. It must then either contain $\pm z$ for all $z \in \Lambda_{24}$ with $|z|^{2}=4$ and $\langle x, z\rangle=1$, in which case it is simply all the minimal vectors in $\Lambda_{24}$, or it must contain all the vectors $\pm(x / 2-z)$ for such $z$. (If it contains one of the points $\pm z$, then it contains all of them because any one of them together with $L$ spans $\Lambda_{24}$ and hence the orthogonal transformation would be 
a symmetry of $\Lambda_{24}$.) One can check as follows that the second possibility is indeed a valid kissing configuration about 0 . If $r$ denotes reflection in the hyperplane through the origin orthogonal to $x$, then the map $z \mapsto-r(z)$ preserves $L$, maps $x$ to $x$, and interchanges the two kissing configurations.

We have shown that there are only two distinct kissing configurations about 0 that both contain $x$ and the 4600 common neighbors. Fortunately, only the first can occur in the sphere packing. Suppose the kissing configuration about 0 contained $x / 2-z$ with $z \in \Lambda_{24},|z|^{2}=4$, and $\langle x, z\rangle=1$. Because $x$ is good, its kissing configuration contains the point $x-z$, which is so close to $x / 2-z$ that the spheres centered at those points would overlap. That contradiction rules out the second case, so 0 must be good as well, which completes the proof.

Under Conjecture 9.4, let $h_{s}$ prove a sharp bound for $f(r)=1 / r^{s}$. It seems likely that, when suitably rescaled, $h_{s}$ converges as $s \rightarrow \infty$ to a function that proves a sharp bound in the setting of $\mathrm{CE}$. (One can check that this holds for the functions constructed below when $n=1$.) That would put the mysterious functions from that paper into a broader context and exhibit them as limits of families of functions.

We have not been able to prove Conjecture 9.4, but we can prove the onedimensional analogue. Of course, minimizing potential energy in $\mathbb{R}^{1}$ is completely trivial, so this result has no interesting geometric consequences. However, it is not obvious that Proposition 9.3 proves a sharp bound even in this trivial case, so we view its sharpness as an argument in favor of Conjecture 9.4,

Proposition 9.6. Let $f:(0, \infty) \rightarrow \mathbb{R}$ be completely monotonic and satisfy $f(x)=$ $O\left(|x|^{-1 / 2-\varepsilon}\right)$ for some $\varepsilon>0$ as $|x| \rightarrow \infty$. Then there exists a function $h: \mathbb{R} \rightarrow \mathbb{R}$ such that $h(x) \leq f\left(x^{2}\right)$ for all $x \in \mathbb{R} \backslash\{0\}, h$ is the Fourier transform of a continuous function $\widehat{h}: \mathbb{R} \rightarrow \mathbb{R}$ with support in $[-1,1], \widehat{h}(t) \geq 0$ for all $t \in \mathbb{R}$, and

$$
\widehat{h}(0)-h(0)=\sum_{k \in \mathbb{Z} \backslash\{0\}} f\left(k^{2}\right) .
$$

For notational convenience, define $\tilde{f}(x)=f\left(x^{2}\right)$ for $x \in \mathbb{R} \backslash\{0\}$ (and let $\tilde{f}^{\prime}(x)=$ $2 x f^{\prime}\left(x^{2}\right)$ denote its derivative). Then we can take

$$
\begin{aligned}
h(x)= & \sum_{k \in \mathbb{Z} \backslash\{0\}} \tilde{f}(k)\left(\frac{\sin \pi(x-k)}{\pi(x-k)}\right)^{2}+\sum_{k \in \mathbb{Z} \backslash\{0\}} \widetilde{f}^{\prime}(k) \frac{(\sin \pi(x-k))^{2}}{\pi^{2}(x-k)} \\
& -2\left(\frac{\sin \pi x}{\pi x}\right)^{2} \sum_{k=1}^{\infty}\left(\widetilde{f}(k)+k \widetilde{f}^{\prime}(k)\right) .
\end{aligned}
$$

(Of course we define $h(x)$ by continuity when $x \in \mathbb{Z}$.) By the mean value theorem and the fact that $f^{\prime}$ is increasing and nonpositive,

$$
\left|\frac{f(x)-f(x / 2)}{x / 2}\right| \geq\left|f^{\prime}(x)\right|
$$

which implies that $\left|x f^{\prime}(x)\right|=O\left(|x|^{-1 / 2-\varepsilon}\right)$; thus, the sum of $k \widetilde{f}^{\prime}(k)$ in the definition of $h$ converges, because $\tilde{f}^{\prime}(k)=2 k f^{\prime}\left(k^{2}\right)$.

We have no reason to think that these functions are the only ones that prove a sharp bound in Proposition 9.3 for $n=1$, but they are the only ones we know how to construct. We prove below that they satisfy the properties listed in Proposition 9.6. 
The construction of $h$ is based on a variant of the Shannon sampling theorem for band-limited functions (see Theorem 9 in $[\mathrm{V}]$ for the particular interpolation formula we use). It is natural to search for a function $h$ such that $\widehat{h}$ vanishes outside $[-1,1]$, by analogy with the one-dimensional construction in Section 5 of $\mathrm{CE}$. One can reconstruct such a function from its values and derivatives at the integers, assuming $h$ is integrable, via

$$
h(x)=\sum_{k \in \mathbb{Z}} h(k)\left(\frac{\sin \pi(x-k)}{\pi(x-k)}\right)^{2}+\sum_{k \in \mathbb{Z}} h^{\prime}(k) \frac{(\sin \pi(x-k))^{2}}{\pi^{2}(x-k)} .
$$

We will not require this general fact, but it is important motivation for the definition of $h$.

It is easy to check from the definition of $h$ that $h(k)=\tilde{f}(k)$ and $h^{\prime}(k)=\tilde{f}^{\prime}(k)$ for $k \in \mathbb{Z} \backslash\{0\}$. These conditions are necessary if $h$ is to prove a sharp bound in Proposition 9.3. We also have $h^{\prime}(0)=0$ (in fact, $h$ is even). However, the value of $h(0)$, which is given by

$$
h(0)=-2 \sum_{k=1}^{\infty}\left(\widetilde{f}(k)+k \widetilde{f}^{\prime}(k)\right)
$$

is more mysterious. Setting $h(0)=\tilde{f}(0)$ would make no sense, because $\tilde{f}(0)=f(0)$, which is frequently undefined. The value we have chosen for $h(0)$ makes $h$ have the right properties, but we know no motivation for it that does not rely on some calculation.

Because

$$
\left(\frac{\sin \pi x}{\pi x}\right)^{2}=\int_{-1}^{1}(1-|t|) e^{2 \pi i t x} d t
$$

and

$$
\frac{(\sin \pi x)^{2}}{\pi^{2} x}=\int_{-1}^{1} \frac{\operatorname{sgn} t}{2 \pi i} e^{2 \pi i t x} d t
$$

$h$ is the Fourier transform of a function $\widehat{h}$ supported on $[-1,1]$. Because $h$ is even, $\widehat{h}$ is also even, so it suffices to compute $\widehat{h}(t)$ for $t \in[0,1]$. (Of course the simplification is inessential, but it avoids absolute value signs.) For such $t$, one finds that

$$
\begin{aligned}
\widehat{h}(t)= & 2(1-t) \sum_{k=1}^{\infty} \widetilde{f}(k) \cos 2 \pi k t-\frac{1}{\pi} \sum_{k=1}^{\infty} \widetilde{f}^{\prime}(k) \sin 2 \pi k t \\
& -2(1-t) \sum_{k=1}^{\infty}\left(\tilde{f}(k)+k \tilde{f}^{\prime}(k)\right) .
\end{aligned}
$$

One motivation for the value of $h(0)$ is that if one formally computes $\widehat{h}^{\prime}(1)$, one gets the answer 0 . However, we do not require this fact and make no attempt to justify it.

To complete the proof of Proposition 9.6. we must prove that $h(x) \leq f\left(x^{2}\right)$ for all $x \neq 0$ and $\widehat{h}(t) \geq 0$ for all $t$. One potential approach is to reduce to the case of $f(x)=e^{-c x}$ with $c>0$ : by Bernstein's theorem (Theorem 12b in Wid, p. 161]), there exists a weakly increasing function $\alpha:[0, \infty) \rightarrow \mathbb{R}$ such that for all $x>0$,

$$
f(x)=\int_{0}^{\infty} e^{-c x} d \alpha(c) .
$$


We can thus assume without loss of generality that $f(x)=e^{-c x}$ with $c>0$. (There is no contribution from $c=0$ because if there were, then $f$ would not decay at infinity.) To see that the inequalities we need are preserved under this reduction, we must check that integration over $c$ commutes with the sums over $k$ in the formulas for $h$ and $\widehat{h}$. That follows from Fubini's theorem.

We have not fully carried out this approach, but it is likely possible. For the less general case of $f(x)=e^{-c \sqrt{x}}$, which corresponds to the special case when not only $f$ but also $x \mapsto f\left(x^{2}\right)$ is completely monotonic, the inequality $h(x) \leq f\left(x^{2}\right)$ is very nearly proved in Theorem 8 of [GV], which constructs similar functions to solve a related optimization problem. That paper does not contain precisely the inequality we need, but a simple modification of the techniques used there suffices, and one can furthermore prove that $\widehat{h}(t) \geq 0$ by computing $\widehat{h}$ explicitly and manipulating it. This proof suffices for potential functions $f$ such that $x \mapsto f\left(x^{2}\right)$ is completely monotonic, but it is somewhat complicated and unilluminating.

Here we check these inequalities (and hence prove Proposition 9.6) via a more conceptual approach, by proving that they follow from the corresponding assertions about codes on $S^{1}$ by taking limits. Consider $N$ points on $S^{1}$, under the completely monotonic potential function $g$; we will define $g$ in terms of $f$ shortly. In the proof of Theorem 1.2, we constructed an auxiliary function $h_{N, g}$ that proved a sharp bound for the $g$-potential energy in Proposition 4.1. When $N$ is odd, one can write it down explicitly as follows. Define $F(t)=\left(T_{N}(t)-1\right) /(t-1)$, where $T_{N}$ is the $N$-th Chebyshev polynomial, given by $T_{N}(\cos \theta)=\cos N \theta$. (The roots of $F$ are the inner products other than 1 in the regular $N$-gon.)

Lemma 9.7. When $N$ is odd,

$$
h_{N, g}(t)=\frac{F(t)}{N^{2}} \sum_{r: F(r)=0} \frac{a_{r}+b_{r}(t-r)}{(t-r)^{2}},
$$

where

$$
a_{r}=2(1-r)^{2}(1+r) g(2-2 r)
$$

and

$$
b_{r}=-4(1-r)^{2}(1+r) g^{\prime}(2-2 r)-2(1-r)(1+2 r) g(2-2 r) .
$$

The sum is over all roots $r$ of $F$, each included only once despite the fact that they are double roots. The proof of this formula amounts to verifying that the right side is the Hermite interpolation of $t \mapsto g(2-2 t)$ to second order at the roots of $F$, which is a straightforward calculation. (The case of even $N$ differs slightly because one must treat the single root $r=-1$ of $F$ differently. We will assume without loss of generality that $N$ is odd, but the even case can easily be handled by the same techniques.)

We define $g$ by

$$
g(x)=f\left(N^{2} x /\left(4 \pi^{2}\right)\right) .
$$

The motivation is that as $N \rightarrow \infty$,

$$
g(2-2 \cos (2 \pi x / N))=\widetilde{f}((N / \pi) \sin (\pi x / N)) \rightarrow \widetilde{f}(x),
$$

which suggests the following lemma. 
Lemma 9.8. For $g(x)=f\left(N^{2} x /\left(4 \pi^{2}\right)\right)$, we have

$$
\lim _{N \rightarrow \infty} h_{N, g}(\cos (2 \pi x / N))=h(x)
$$

as $N \rightarrow \infty$ with $N$ odd.

The same is also true for $N$ even, but we will not need it. Note that Lemma 9.8 makes no assertion as to the uniformity of the convergence (pointwise convergence will suffice for our purposes).

Proof. At a formal level, it follows from taking limits term by term and using

$$
\lim _{N \rightarrow \infty} \frac{g^{\prime}(2-2 \cos (2 \pi x / N))}{(N /(2 \pi))^{2}}=\frac{\tilde{f}^{\prime}(x)}{2 x}
$$

and

$$
\lim _{N \rightarrow \infty} \frac{F(\cos (2 \pi x / N))}{N^{2}}=\left(\frac{\sin \pi x}{\pi x}\right)^{2}
$$

that $h_{N, g}(\cos (2 \pi x / N))$ converges to

$$
\left(\frac{\sin \pi x}{\pi x}\right)^{2} \sum_{k=1}^{\infty}\left(\widetilde{f}(k)\left(\frac{2 k x-k^{2}}{(x-k)^{2}}-\frac{2 k x+k^{2}}{(x+k)^{2}}\right)+\widetilde{f}^{\prime}(k)\left(\frac{k^{2}}{x-k}-\frac{k^{2}}{x+k}\right)\right),
$$

which is equal to $h(x)$. (The roots of $F$ are of the form $\cos (2 \pi k / N)$, which is how the sum over $r$ becomes a sum over $k$.) To prove rigorously that

$$
\lim _{N \rightarrow \infty} h_{N, g}(\cos (2 \pi x / N))=h(x),
$$

one must justify taking the limit term by term.

To do so, we will apply the dominated convergence theorem. Set

$$
r=\cos (2 \pi k / N)=1-2 \sin ^{2}(\pi k / N)
$$

for $0<k<N / 2$, and turn the sum over $r$ in the formula for $h_{N, g}(t)$ into a sum over all $k>0$ by including zero terms for all larger $k$. Let $t=1-2 \sin ^{2}(\pi x / N)$. We will prove that the $k$-th term in the sum is $O\left(k^{-1-2 \varepsilon}\right)$ in absolute value, where the implicit constant is independent of $N$. (Of course this calculation uses $f(x)=$ $O\left(|x|^{-1 / 2-\varepsilon}\right)$ and $f^{\prime}(x)=O\left(|x|^{-3 / 2-\varepsilon}\right)$.) Because

$$
\sum_{k=1}^{\infty} k^{-1-2 \varepsilon}
$$

converges, taking limits term by term is justified by the dominated convergence theorem.

One can calculate that the $k$-th term is given by

$$
\frac{a_{r}+b_{r}(t-r)}{(t-r)^{2}}=\frac{2\left(2 s_{k}^{2}-1+3\left(s_{x} / s_{k}\right)^{2}-4 s_{x}^{2}\right)}{\left(1-\left(s_{x} / s_{k}\right)^{2}\right)^{2}} f_{k}-\frac{4 N^{2} s_{k}^{2}\left(1-s_{k}^{2}\right)}{\pi^{2}\left(1-\left(s_{x} / s_{k}\right)^{2}\right)} f_{k}^{\prime},
$$

where to save space we write

and

$$
\begin{gathered}
s_{x}=\sin (\pi x / N), \\
s_{k}=\sin (\pi k / N), \\
f_{k}=f\left((N / \pi)^{2} \sin ^{2}(\pi k / N)\right),
\end{gathered}
$$

$$
f_{k}^{\prime}=f^{\prime}\left((N / \pi)^{2} \sin ^{2}(\pi k / N)\right) .
$$


We always have $0<k<N / 2$, and for fixed $x$ we have $0<x<N / 2$ whenever $N$ is sufficiently large relative to $x$. It follows that $s_{k}$ and $s_{x}$ are always within fixed constant factors of $\pi k / N$ and $\pi x / N$, respectively. If $k$ is also sufficiently large relative to $x$, then examining (9.2) and applying $N^{2} s_{k}^{2}=O\left(k^{2}\right)$ shows that the right side is $O\left(k^{-1-2 \varepsilon}\right)$ in absolute value, where the implicit constant is independent of $N$.

The desired inequalities for $h$ and $\widehat{h}$ now follow easily. For each $N$,

$$
h_{N, g}(\cos (2 \pi x / N)) \leq g(2-2 \cos (2 \pi x / N)),
$$

so it follows in the limit as $N \rightarrow \infty$ that

$$
h(x) \leq \widetilde{f}(x)
$$

as desired. For the other inequality, we apply Bochner's theorem, which says that a continuous function $h$ on $\mathbb{R}$ is the Fourier transform of a nonnegative, $L^{1}$ function (which is of course uniquely determined by $h$, up to a set of measure 0 ) if and only if $h$ is positive definite, in the sense that for all $x_{1}, \ldots, x_{\ell} \in \mathbb{R}$, the $\ell \times \ell$ matrix whose $i, j$ entry is $h\left(x_{i}-x_{j}\right)$ is positive semidefinite. (See [St, p. 124] or [R, p. 303].) That property follows immediately from the corresponding property of $h_{N, g}$, because the set of positive-semidefinite matrices is closed. This completes the proof of Proposition 9.6 .

\section{ACKNOWLEDGEMENTS}

We thank Eiichi Bannai, Charles Doran, Noam Elkies, Gabriele Nebe, Andreas Strömbergsson, Frank Vallentin, and Günter Ziegler for their valuable comments and suggestions on our manuscript.

\section{Appendix A. Uniqueness of SPHERICAL CODES}

Uniqueness of the $N$-gon is trivial. For the simplex, cross polytope, icosahedron, and 600-cell, uniqueness follows from analyzing the case of equality in the Bőröczky bound (see [Bö, p. 260], or see [BD for another proof for the 600-cell). For the $(24,196560,1 / 2),(23,4600,1 / 3),(8,240,1 / 2)$, and $(7,56,1 / 3)$ spherical codes, uniqueness was proved by Bannai and Sloane in [BS] (see also Chapter 14 of [CS]). For the $(6,27,1 / 4),(5,16,1 / 5),(22,275,1 / 6),(21,162,1 / 7)$, and $(22,100,1 / 11)$ codes, uniqueness follows from results in the theory of strongly regular graphs. In each case there are only two distinct inner products other than 1 , so one can form a graph out of the vertices by making edges correspond to one particular inner product (one obtains one of two complementary graphs, which encode the same information). Using Theorem 7.4 and Lemma 7.3 from [DGS one proves that these graphs are strongly regular and determines their parameters. In each case there are unique strongly regular graphs with these parameters. For the $(6,27,1 / 4)$ and $(5,16,1 / 5)$ codes, see Theorem 10.6.4 and Lemma 10.9.4 in GR; the remaining cases are dealt with in CGS. It follows that the Gram matrices of the corresponding point configurations are uniquely determined, so the configurations are determined up to orthogonal transformations.

For the $(23,552,1 / 5)$ code, uniqueness follows from the uniqueness of the regular two-graph on 276 vertices. In any $(23,552,1 / 5)$ code, the linear programming bounds show that only the inner products -1 and $\pm 1 / 5$ occur. Such a code thus gives rise to an arrangement of 276 equiangular lines in $\mathbb{R}^{23}$, which corresponds 
to a regular two-graph on 276 vertices. (See Chapter 11 of GR for background on two-graphs and equiangular line arrangements.) Goethals and Seidel proved in GS that it is unique, which implies the uniqueness of the $(23,552,1 / 5)$ code.

The last remaining case is the $(22,891,1 / 4)$ spherical code. A proof of its uniqueness is implicit in $[\mathrm{Cu}$ (based on results from $[\mathrm{SY}]$ ). For an alternative proof using the techniques of Bannai and Sloane, see [CK3].

\section{REFERENCES}

[An1] N. N. Andreev, An extremal property of the icosahedron, East J. Approx. 2 (1996), 459-462. MR:1426716 (97m:52022)

[An2] N. N. Andreev, Location of points on a sphere with minimal energy (Russian), Tr. Mat. Inst. Steklova 219 (1997), 27-31; translation in Proc. Steklov Inst. Math. 219 (1997), 20-24. MR 1642295 (99i:52019)

[An3] N. N. Andreev, A spherical code (Russian), Uspekhi Mat. Nauk 54 (1999), 255-256; translation in Russian Math. Surveys 54 (1999), 251-253. MR.1706807

[AAR] G. Andrews, R. Askey, and R. Roy, Special Functions, Encyclopedia of Mathematics and its Applications 71, Cambridge University Press, Cambridge, 1999. MR1688958 (2000g:33001)

[As] R. Askey, Orthogonal Polynomials and Special Functions, Society for Industrial and Applied Mathematics, Philadelphia, Pennsylvania, 1975. MR0481145 (58:1288)

[Ba] J. Baez, The octonions, Bull. Amer. Math. Soc. (N.S.) 39 (2002), 145-205, arXiv: math.RA/0105155; see also errata in Bull. Amer. Math. Soc. 42 (2005), 213. MR.1886087 (2003f:17003) MR2132837

[BMV] E. Bannai, A. Munemasa, and B. Venkov, The nonexistence of certain tight spherical designs, Algebra i Analiz 16 (2004), 1-23. MR2090848 (2005e:05022)

[BS] E. Bannai and N. J. A. Sloane, Uniqueness of certain spherical codes, Canad. J. Math. 33 (1981), no. 2, 437-449. MR0617634 (83a:94020)

[Bő] K. Bőrőczky, Packing of spheres in spaces of constant curvature, Acta Math. Acad. Sci. Hung. 32 (1978), 243-261. MR0512399 (80h:52014)

[BöJr] K. Börőczky, Jr., Finite packing and covering, Cambridge Tracts in Mathematics 154, Cambridge University Press, Cambridge, 2004. MR2078625 (2005g:52045)

[BD] P. Boyvalenkov and D. Danev, Uniqueness of the 120-point spherical 11-design in four dimensions, Arch. Math. (Basel) 77 (2001), no. 4, 360-368. MR 1853553 (2002f:05045)

[CGS] P. J. Cameron, J. M. Goethals, and J. J. Seidel, Strongly regular graphs having strongly regular subconstituents, J. Algebra 55 (1978), 257-280. MR.0523457 (81d:05034)

[Co] H. Cohn, New upper bounds on sphere packings II, Geom. Topol. 6 (2002), 329-353, arXiv:math.MG/0110010. MR 1914571(2004b:52032)

[CE] H. Cohn and N. D. Elkies, New upper bounds on sphere packings I, Ann. of Math. 157 (2003), 689-714, arXiv:math.MG/0110009. MR1973059(2004b:11096)

[CK1] H. Cohn and A. Kumar, Optimality and uniqueness of the Leech lattice among lattices, preprint, 2003, arXiv:math.MG/0403263.

[CK2] H. Cohn and A. Kumar, The densest lattice in twenty-four dimensions, Electron. Res. Announc. Amer. Math. Soc. 10 (2004), 58-67, arXiv:math.MG/0408174. MR2075897 (2005e:11089)

[CK3] H. Cohn and A. Kumar, Uniqueness of the $(22,891,1 / 4)$ spherical code, preprint, 2004, arXiv:math.MG/0607448.

[CCEK] H. Cohn, J. H. Conway, N. D. Elkies, and A. Kumar, The $D_{4}$ root system is not universally optimal, preprint, 2004, arXiv:math.MG/0607447.

[CHS] J. Conway, R. Hardin, and N. J. A. Sloane, Packing lines, planes, etc.: packings in Grassmannian spaces, Experiment. Math. 5 (1996), 139-159. MR1418961(98a:52029)

[CS] J. Conway and N. J. A. Sloane, Sphere Packings, Lattices and Groups, third edition, Grundlehren der Mathematischen Wissenschaften 290, Springer-Verlag, New York, 1999. MR 1662447 (2000b:11077)

$[\mathrm{Cu}] \quad \mathrm{H}$. Cuypers, A note on the tight spherical 7-design in $\mathbb{R}^{23}$ and 5-design in $\mathbb{R}^{7}$, Des. Codes Cryptogr. 34 (2005), 333-337. MR2128339 (2005k:05058) 
[D] P. J. Davis, Interpolation and Approximation, Blaisdell Publishing Company, New York, 1963. MR0157156 (28:393)

[DGS] P. Delsarte, J. Goethals, and J. Seidel, Spherical codes and designs, Geometriae Dedicata 6 (1977), 363-388. MR0485471 (58:5302)

[Eb] W. Ebeling, Lattices and codes. A course partially based on lectures by F. Hirzebruch, second edition, Advanced Lectures in Mathematics, Friedr. Vieweg \& Sohn, Braunschweig, 2002. MR.1938666 (2003i:11093)

[El] N. Elkies, Optimal codes in homogeneous spaces, unpublished manuscript, 1995.

[Gan] R. Gangolli, Positive definite kernels on homogeneous spaces and certain stochastic processes related to Lévy's Brownian motion of several parameters, Ann. Inst. H. Poincaré Sect. B (N.S.) 3 (1967), 121-226. MR0215331 (35:6172)

[Gas] G. Gasper, Linearization of the product of Jacobi polynomials I, Canad. J. Math. 22 (1970), 171-175. MR0257433(41:2084)

[GR] C. Godsil and G. Royle, Algebraic Graph Theory, Graduate Texts in Mathematics 207, Springer-Verlag, New York, 2001. MR1829620(2002f:05002)

[GS] J. Goethals and J. Seidel, The regular two-graph on 276 vertices, Discrete Math. 12 (1975), 143-158. MR0384597 (52:5471)

[GV] S. W. Graham and J. D. Vaaler, A class of extremal functions for the Fourier transform, Trans. Amer. Math. Soc. 265 (1981), no. 1, 283-302. MR0607121 (82i:42008)

$[\mathrm{H}] \quad$ R. Hartshorne, Algebraic Geometry, Graduate Texts in Mathematics 52, SpringerVerlag, New York, 1977. MR0463157 (57:3116)

[HJ] R. A. Horn and C. R. Johnson, Matrix Analysis, Cambridge University Press, 1985. MR.0832183 (87e:15001)

[KL] G. A. Kabatiansky and V. I. Levenshtein, Bounds for packings on a sphere and in space (Russian), Problemy Peredači Informacii 14 (1978), 3-25; translation in Problems of Information Transmission 14 (1978), no. 1, 1-17. MR0514023 (58:24018)

[KY1] A. V. Kolushov and V. A. Yudin, On the Korkin-Zolotarev construction (Russian), Diskret. Mat. 6 (1994), 155-157; translation in Discrete Math. Appl. 4 (1994), 143-146. MR.1273240 (95j:52033)

[KY2] A. V. Kolushov and V. A. Yudin, Extremal dispositions of points on the sphere, Anal. Math. 23 (1997), 25-34. MR1630001 (99f:41039)

[Lee] J. Leech, Equilibrium of sets of particles on a sphere, Math. Gaz. 41 (1957), 81-90. MR.0086325 (19:165b)

[Lev1] V. I. Levenshtein, On bounds for packings in n-dimensional Euclidean space (Russian), Dokl. Akad. Nauk SSSR 245 (1979), 1299-1303; translation in Soviet Math. Dokl. 20 (1979), 417-421. MR0529659(80d:52017)

[Lev2] V. I. Levenshtein, Designs as maximum codes in polynomial metric spaces, Acta Appl. Math. 29 (1992), 1-82. MR1192833 (93j:05012)

[Lev3] V. I. Levenshtein, Universal bounds for codes and designs, in Handbook of Coding Theory, Vol. I, North-Holland, Amsterdam, 1998, pages 499-648. MR 1667942

[Man] Yu. I. Manin, Cubic Forms: Algebra, Geometry, Arithmetic, second edition, NorthHolland Mathematical Library 4, North-Holland, Amsterdam, 1986. MR0833513 (87d:11037)

[Mar] J. Martinet, Perfect lattices in Euclidean spaces, Grundlehren der Mathematischen Wissenschaften 327, Springer-Verlag, Berlin, 2003. MR.1957723(2003m:11099)

[MRRW] R. J. McEliece, E. R. Rodemich, H. C. Rumsey, Jr., and L. R. Welch, New upper bounds on the rate of a code via the Delsarte-MacWilliams inequalities, IEEE Trans. Information Theory 23 (1977), 157-166. MR0439403 (55:12296)

[Mo] H. Montgomery, Minimal theta functions, Glasgow Math. J. 30 (1988), 75-85. MR0925561 (89d:11029)

[OS] A. M. Odlyzko and N. J. A. Sloane, New bounds on the number of unit spheres that can touch a unit sphere in $n$ dimensions, J. Combin. Theory Ser. A 26 (1979), 210-214. MR.0530296 (81d:52010)

[PS] G. Pólya and G. Szegö, Problems and theorems in analysis, Vol. II, revised and enlarged translation by C. E. Billigheimer of the fourth German edition, Springer-Verlag, New York-Heidelberg, 1976. MR0465631 (57:5529)

[R] W. Rudin, Functional Analysis, second edition, McGraw-Hall, New York, 1991. MR $1157815(92 \mathrm{k}: 46001)$ 
[SS] P. Sarnak and A. Strömbergsson, Minima of Epstein's zeta function and heights of flat tori, Invent. Math. 165 (2006), 115-151. MR.2221138

[Sch] I. J. Schoenberg, Positive definite functions on spheres, Duke Math. J. 9 (1942), 96-108. MR.0005922 (3:232c)

[SchW] K. Schütte and B. L. van der Waerden, Auf welcher Kugel haben 5, 6, 7, 8 oder 9 Punkte mit Mindestabstand 1 Platz?, Math. Ann. 123 (1951), 96-124. MR0042150(13:61e)

[SZ] P. D. Seymour and T. Zaslavsky, Averaging sets: a generalization of mean values and spherical designs, Adv. in Math. 52 (1984), 213-240. MR0744857 (85m:05031)

[SY] E. Shult and A. Yanushka, Near n-gons and line systems, Geom. Dedicata 9 (1980), 1-72. MR 0566437 (82b:51018)

[Si] B. Simon, Orthogonal polynomials on the unit circle, Part 1: Classical theory, American Mathematical Society Colloquium Publications 54, American Mathematical Society, Providence, RI, 2005. MR 2105088 (2006a:42002a)

[Sl] N. J. A. Sloane, with the collaboration of R. H. Hardin, W. D. Smith, and others, Tables of spherical codes, published electronically at http://www.research.att.com/ ${ }^{\sim}$ jas/ packings/.

[StW] E. M. Stein and G. Weiss, Introduction to Fourier analysis on Euclidean spaces, Princeton Mathematical Series 32, Princeton University Press, Princeton, New Jersey, 1971. MR.0304972(46:4102)

[St] R. Strichartz, A Guide to Distribution Theory and Fourier Transforms, CRC Press, Boca Raton, Florida, 1994. MR1276724 (95f:42001)

[Sz] G. Szegö, Orthogonal Polynomials, fourth edition, AMS Colloquium Publications 23, American Mathematical Society, Providence, Rhode Island, 1975. MR0372517|(51:8724)

[T] J. Tits, Ovoïdes et groupes de Suzuki, Arch. Math. 13 (1962), 187-198. MR0140572 $(25: 3990)$

[V] J. D. Vaaler, Some extremal functions in Fourier analysis, Bull. Amer. Math. Soc. (N.S.) 12 (1985), 183-216. MR0776471 (86g:42005)

[Wa] H. C. Wang, Two-point homogeneous spaces, Ann. Math. 55 (1952), 177-191. MR.0047345 (13:863a)

[Wid] D. V. Widder, The Laplace Transform, Princeton University Press, Princeton, New Jersey, 1941. MR0005923 (3:232d)

[Wil] R. A. Wilson, Vector stabilizers and subgroups of Leech lattice groups, J. Algebra 127 (1989), 387-408. MR.1028461 (91g:20020)

[Y] V. A. Yudin, Minimum potential energy of a point system of charges (Russian), Diskret. Mat. 4 (1992), 115-121; translation in Discrete Math. Appl. 3 (1993), 75-81. MR 1181534(93f:31008)

Microsoft Research, One Microsoft Way, Redmond, Washington 98052-6399

E-mail address: cohn@microsoft.com

Department of Mathematics, Harvard University, Cambridge, Massachusetts 02138

E-mail address: abhinav@math.harvard.edu

Current address: Microsoft Research, One Microsoft Way, Redmond, Washington 98052-6399

E-mail address: abhinavk@microsoft.com 